\title{
Quinazolin-4-one derivatives lacking toxicity-producing attributes as glucokinase activators: design, synthesis, molecular docking, and in-silico ADMET prediction
}

Saurabh C. Khadse ${ }^{1}$, Nikhil D. Amnerkar ${ }^{2 *}$ (D, Manasi U. Dave ${ }^{1}$, Deepak K. Lokwani ${ }^{1}$, Ravindra R. Patil ${ }^{1}$, Vinod G. Ugale ${ }^{1}$, Nitin B. Charbe ${ }^{3,4}$ and Vivekanand A. Chatpalliwar ${ }^{5}$

\begin{abstract}
Background: A small library of quinazolin-4-one clubbed thiazole acetates/acetamides lacking toxicity-producing functionalities was designed, synthesized, and evaluated for antidiabetic potential as glucokinase activators (GKA). Molecular docking studies were done in the allosteric site of the human glucokinase (PDB ID: 1V4S) enzyme to assess the binding mode and interactions of synthesized hits for best-fit conformations. All the compounds were evaluated by in vitro enzymatic assay for GK activation.

Results: Data showed that compounds $3\left(E C_{50}=632 \mathrm{nM}\right)$ and $4\left(E C_{50}=516 \mathrm{nM}\right)$ showed maximum GK activation compared to the standards RO-281675 and piragliatin. Based on the results of the in vitro enzyme assay, docking studies, and substitution pattern, selected compounds were tested for their glucose-lowering effect in vivo by oral glucose tolerance test (OGTT) in normal rats. Compounds $3(133 \mathrm{mg} / \mathrm{dL})$ and $4(135 \mathrm{mg} / \mathrm{dL})$ exhibited prominent activity by lowering the glucose level to almost normal, eliciting the results in parallel to enzyme assay and docking studies. Binding free energy, hydrogen bonding, and $\pi-\pi$ interactions of most active quinazolin-4-one derivatives 3 and 4 with key amino acid residues of the 1V4S enzyme were studied precisely. Preliminary in-silico absorption, distribution, metabolism, excretion, and toxicity (ADMET) prediction was carried out using SwissADME and PreADMET online software which revealed that all the compounds have the potential to become orally active antidiabetic agents as they obeyed Lipinski's rule of five.

Conclusion: The results revealed that the designed lead could be significant for the strategic design of safe, effective, and orally bioavailable quinazolinone derivatives as glucokinase activators.
\end{abstract}

Keywords: Glucokinase activator, Glucokinase, Quinazolinones, Docking

\section{Background}

Glucokinase (GK) is a glucose-phosphorylating enzyme that converts glucose to glucose-6-phosphate. It is known as an exceptional drug target for antidiabetic therapy due to its glucose sensor role in regulating the threshold for glucose-stimulated insulin secretion from pancreatic $\beta$-cells, rate-controlling glucose clearance and glycogen synthesis in the live r[1]. Thus, GK activators

\footnotetext{
* Correspondence: nikhilamnerkar@gmail.com

${ }^{2}$ Adv. V. R. Manohar Institute of Diploma in Pharmacy, Wanadongri, Hingna

Road, Nagpur 441110, India

Full list of author information is available at the end of the article
}

(GKAs) are expected to act as hypoglycemic agents by performing dual functions of increasing glucose uptake in the liver and enhancing insulin secretion by pancreatic $\beta$-cell $s[2]$. The discovery of the allosteric activator site in glucokinase and the development of drugs that interact with this site are important milestones in research related to glucose homeostasis and diabetes mellitu s[3, 4]. Serendipitously discovered GKAs including RO-28-1675 [5], piragliatin [6], LY-2121260 [7], benzene-centered GKA [8], PSN-GK1 [9], GKA50 [10], and GKA60 [11] by various pharmaceutical companies led to the discovery of several amides such as 
benzamides, acetamides, and propanamide, that act as crucial pharmacophore to bind to the allosteric site of GK. These molecules also possess primary structural attributes such as urea, heterocycles consisting of nitrogen, sulfur, and oxygen that promised to lower the blood sugar level through GK activation [12]. However, these molecules failed to pass beyond the trials due to the toxicities associated with them [4].

The X-ray crystallography studies have established the key features of the ligand structure that interact with amino acid residues and bind in the allosteric site of GK and maintain its conformation in the active mode [13]. The role of thiazole ring, a primary aromatic amino group and with a structural feature like cyclopentane/ one and pyridine acid in these potent ligands was found crucial for interaction with the allosteric site of GK. However, unfortunately, some of these structural features stated above lead to the production of toxic metabolites. Literature survey revealed that pyridinic acid moiety elicits testicular toxicity by functioning as retinoic acid receptor antagonist [10, 14]. Likewise, the oxidative metabolism of thiazole group forms toxic thiourea metabolite that was deemed responsible for potential cardiovascular risk and reversible hepatic lipidosis $[15,16]$. Monosubstitution on the C-4 or C5 position of thiazole ring provided a good balance between GK activation potency and oral exposure whereas disubstitution was reported to result in either loss of activity or mild activation of GK except for the cyclization which results in compounds with potent GK activation [12, 17, 18]. Moreover, primary aromatic amines like aniline compounds show the potential risk of mutagenicit $y[8,19]$.

The heterocyclic lead, quinazoline, emerges as a modified aniline analog on optimizing the toxicity imparting primary aromatic amino group. An insight into the binding of 4,6-disubstituted quinazoline ligand on interaction with GK revealed that the nitrogen atom at the 1-position of the quinazoline core interacted with Tyr215 of GK, acting as hydrogen bond donor and the nitrogen atom of the thiazole ring and thiazolamine $\mathrm{NH}$ interact with Arg63 of GK showing good GK-activating property $[8,20]$. Subsequently, not enough literature is available on this line of research. Taking into consideration these ligand-GK interaction data, we planned to design new GKA scaffold from the existing molecular leads keeping in mind the following points: (a) residues should show favorable interactions with GK enzyme, (b) should overcome toxic liabilities, and (c) have the proper balance of potency and physicochemical properties (Fig. 1). Thus, we have synthesized and reported a series of quinazolin-4-one derivatives as the lead for the GK activation.

\section{Methods}

\section{Instrumentation and chemicals}

All chemicals and reagents used were of laboratory grade and procured from Sigma-Aldrich (St. Louis, MO, USA), Rankem (Gurgaon, India), and S.D. Fine Chemicals Pvt Ltd. (Mumbai, India). Melting points were determined by using Analab scientific instrument (Thermocal) by an open capillary method and are uncorrected. Solvents were redistilled and dried as and when required. Reactions were routinely monitored by thin layer chromatography (TLC); aluminum sheets $(2.0-6.0 \mathrm{~cm})$ precoated with silica gel 60 (HF-254, E. Merck, India) were used to perform TLC using solvent system toluene: ethyl acetate (A, 7:3 and B, 8:2; $v / v$ ). Spots were visualized by exposure to iodine vapor or UV light. The IR spectra were recorded on a Shimadzu FTIR 8400S spectrophotometer (Kyoto, Japan) using $\mathrm{KBr}$ optics. ${ }^{1} \mathrm{HNMR}$ (DMSO/ $\mathrm{CDCl}_{3}$ ) and ${ }^{13} \mathrm{CNMR}$ spectra of the synthesized compounds were performed with Bruker Avance-II 400 $\mathrm{MHz}$ spectrometer (Billerica, MA, USA), using tetramethylsilane (TMS) as an internal standard. Mass spectra of the synthesized compounds were performed with WATERS, Q-TOF MICROMASS (Waters, Milford, MA, USA). Elemental analyses were carried out using FLASH EA $1112 \mathrm{CHN}$ analyzer (Thermo Finnigan, Italy) and found within $\pm 0.4 \%$ of theoretical values.

\section{Synthesis}

The reaction of anthranilic acid (1) with acetic anhydride/ propionic anhydride yielded 2-methyl-4H-3,1-benzoxazin-4-one (2)/ 2-ethyl-4H-3,1-benzoxazin-4-one (11) respectively and with benzoyl chloride yielded 2-phenyl4H-3,1-benzoxazin-4-one (20) [21, 22]. The reaction of 2-substituted benzoxazinone $(2,11,20)$ with ethyl-2amino-4-thiazoleacetate yielded ethyl-[2-(2-methyl-4oxoquinazolin-3(4H)-yl)-1,3-thiazol-4-yl]acetate (3), ethyl-[2-(2-ethyl-4-oxoquinazolin-3(4H)-yl)-1,3-thiazol4-yl]acetate (12) and ethyl-[2-(4-oxo-2-phenylquinazolin-3(4H)-yl)-1,3-thiazol-4-yl]acetate (21), respectively. In this reaction, nitrogen nucleophile readily attacks the reactive 4th position of benzoxazinone, followed by ring opening and then closing to generate quinazolinones. The reaction of different aromatic primary amines with 2 -substituted quinazolinone acetate $(3,12,21)$ in dry pyridine yielded the corresponding 2-substituted quinazoline-4-one amide derivatives $(4-10,13-19,22-$ 28) by ammonolysis of esters as portrayed in Scheme 1.

General procedure for synthesis of ethyl [2-(2-substituted-4oxoquinazolin-3(4H)-yl)-1,3-thiazol-4-yl]acetate $(3,12,21)$

The appropriate 2-substituted benzoxazinones (0.01 mol; 2,11 , and 20) were suspended in dry pyridine (15 $\mathrm{mL}$ ) contained in a $100-\mathrm{mL}$ round bottom flask. Ethyl-2amino-4-thiazoleacetate $(0.01 \mathrm{~mol})$ was added to the 


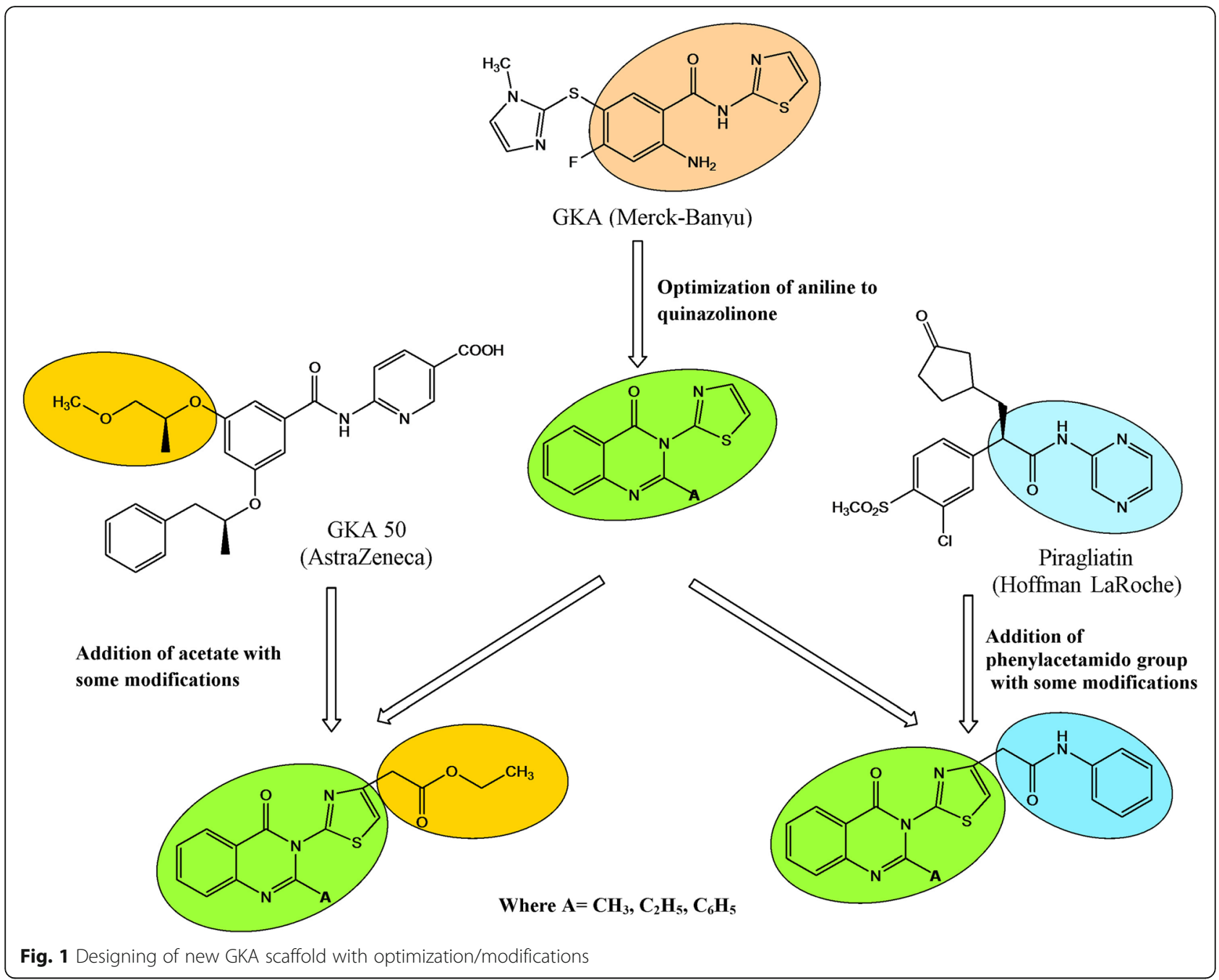

content of the round bottom flask, and the reaction mixture was refluxed for $6-8 \mathrm{~h}$. The reaction mixture was cooled, and excess of pyridine was distilled off under reduced pressure to obtain a yellow-brown color crude product. The products were washed with $0.1 \mathrm{~N} \mathrm{HCl}$ and water, dried, and recrystallized from ethanol.

\section{General procedure for synthesis of 2(2-(2-substituted-4- oxoquinazolin-3-(4H)-yl)-1,3-thiazol-4-yl)-N-substituted acetamide (4-10, 13-19, 22-28)}

Ice cold ethanolic solution of appropriate ethyl [2-(2substituted-4-oxoquinazolin-3(4H)-yl)-1,3-thiazol-4-yl]acetate $(3,12,21)(0.01 \mathrm{~mol})$ was added drop-wise to a mixture of appropriate amines $(0.01 \mathrm{~mol})$ and sodium acetate $(0.01 \mathrm{~mol})$ in dry pyridine $(10-15 \mathrm{~mL})$. The mixture was stirred for 7-10 h maintaining the temperature between $0-5{ }^{\circ} \mathrm{C}$. Then the solution was poured onto crushed ice in a beaker, which was previously acidified with a few drops of hydrochloric acid (5\%). Stirring the resultant product yielded pale yellow to colorless precipitate. The products were washed with $\mathrm{HCl}(0.1 \mathrm{~N})$ and water, dried, and recrystallized from ethanol.

\section{Antidiabetic study}

In vitro GK assay

In vitro $\mathrm{GK}$ assay was performed by using purified human GK. In this method, the GK activity was measured by glucose-6-phosphate dehydrogenase coupled continuously with the spectrophotometric assay. All the compounds were prepared in DMSO, and the assay mixture consists of $25 \mathrm{mM} / \mathrm{L} \mathrm{KCl}, 25 \mathrm{mM} / \mathrm{L}$ 2-(4-(2-hydroxyethyl)piperazin-1-yl)ethane sulfonic acid (HEPES, pH 7.1), $5 \mathrm{mM} / \mathrm{L}$ beta-mercaptoethanol, $1 \mathrm{mM} / \mathrm{L}$ NADP, 1 $\mathrm{mM} / \mathrm{L}$ adenosine triphosphate (ATP), $2.5 \mathrm{mM} / \mathrm{L} \mathrm{MgCl}_{2}$, $2 \mathrm{U} / \mathrm{mL}$ glucose-6-phosphate dehydrogenase, $60 \mathrm{nM} / \mathrm{L}$ GK, and $10 \mathrm{mM}$ glucose. GK activity was measured spectrophotometrically $(340 \mathrm{~nm})$ at room temperature. The $\mathrm{EC}_{50}$ value was calculated from the $\mathrm{OD}$ value at each concentration and used as indices of GK activator potency of the compound. Mean of three readings for 


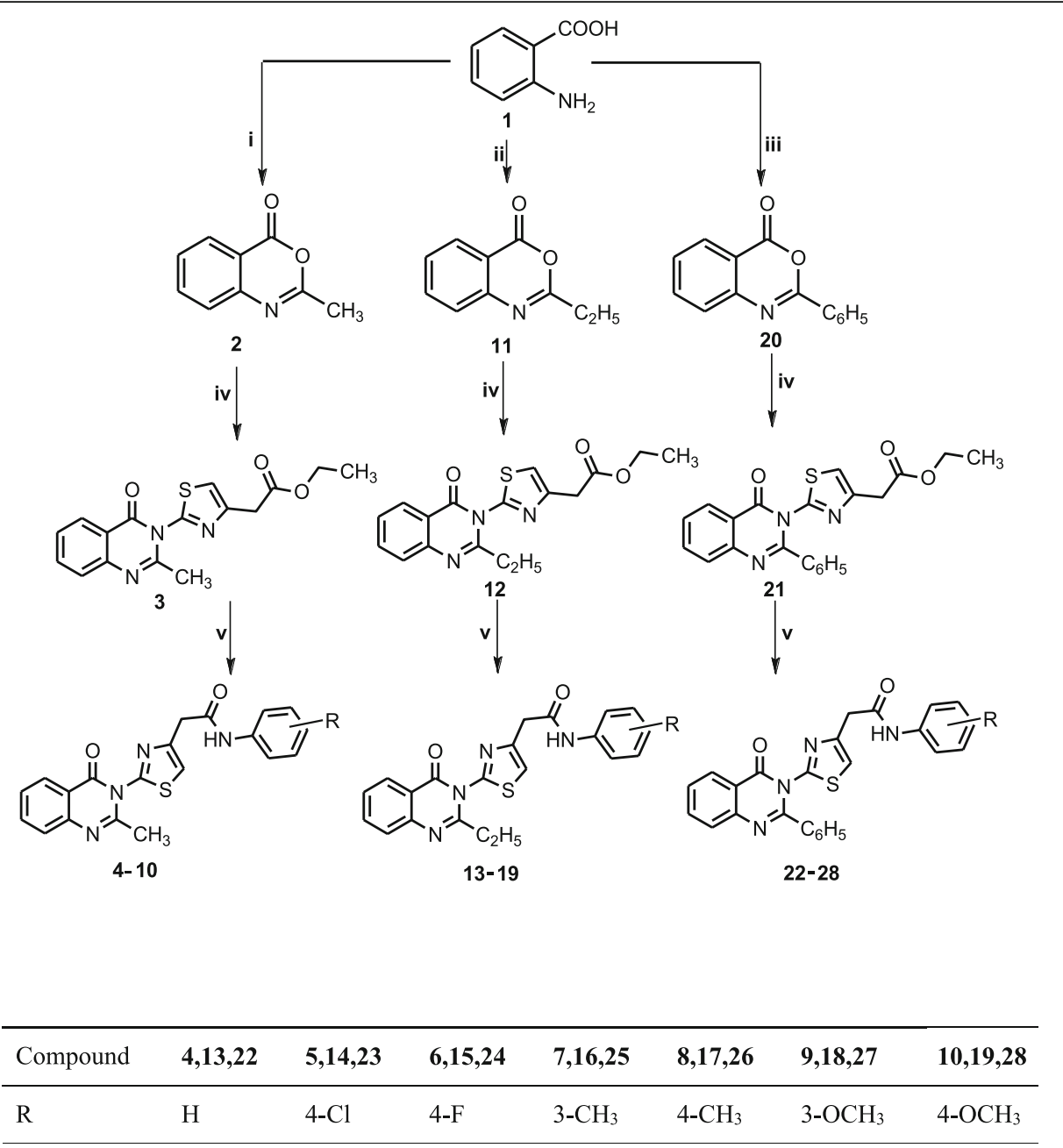

Scheme 1 General scheme for the synthesis of compounds 4-10, 13-19 and 22-28. Reagents and conditions: (i) Acetic anhydride, reflux, 2-3 h, (ii) Propionic anhydride, reflux, 2-3 h, (iii) benzoyl chloride, pyridine, stirring, 1 h, (iv) Ethyl-2-amino-4-thiazoleacetate, pyridine, reflux, 5-6 h, (v) substituted amines, pyridine, sodium acetate, stirring, $0-5{ }^{\circ} \mathrm{C}, 7-10 \mathrm{~h}$.

each test compound and standard were calculated and determined as $\mathrm{EC}_{50}$ values $[23,24]$.

\section{Oral glucose tolerance test (OGTT)}

The rats were procured from our Central Animal House Facility of R. C. Patel Institute of Pharmaceutical Education \& Research (Registered under CPCSEA, India, 651/ $\mathrm{PO} / \mathrm{RcBi} / \mathrm{S} / \mathrm{CPCSEA})$. Male Wistar rats weighing 150$200 \mathrm{~g}$ were subjected to the biological testing. They were housed in polythene cage under standard condition of 12-h light-and-dark cycle at (temperature $22 \pm 2{ }^{\circ} \mathrm{C}$ and humidity $45 \pm 5 \%$ ). The rats were provided with standard pelletized feed (Amrut Rat Feed, Pune, India), and water was made available ad libitum. The employed experimental protocols were as per the ethical principles and guidelines, and approved by the Institutional Animal Ethical Committee constituted for the purpose of control and supervision of experimental animals.
The male Wistar rats $(n=5)$ were fasted overnight before performing the test. The test compounds at a dose of 20 $\mathrm{mg} / \mathrm{kg}$ or vehicle alone $(0.5 \%$ carboxymethyl cellulose solution) were orally administrated to rats, followed by an oral glucose challenge $(2 \mathrm{~g} / \mathrm{kg}) 30 \mathrm{~min}$ later. The plasma glucose concentrations were measured following the glucose challenge $(0,30,60,90$, and $120 \mathrm{~min})$ using a portable glucometer (Accu-Check Active GN03770116) [23-25].

\section{Molecular docking protocol}

All the computational studies were carried out using Glide, version 7.4, Schrodinger, LLC, New York, USA, 2017. The X-ray Crystallographic structure of GK was retrieved from the RCSB Protein Data Bank (PDB) as entry 1V4S [26]. The protein preparation was carried out using Protein Preparation Wizard in Maestro v11.1 in two steps, preparation and refinement and ignoring water molecules. Subsequently, the optimization of 
hydrogen bonds was carried out, followed by energy minimization of protein structure using an OPLS3 force field [27]. 3D structures of designed ligands (quinazolinone derivatives) were prepared using the LigPrep module. The low-energy conformers were generated using an OPLS3 force field. The receptor grid generation panel in Glide module was used to define receptor, and the size of the grid box was fixed so that the ligand with the size of $\leq 20 \AA$ can be docked [28]. The generated low-energy conformers of all ligands were docked into the active site of $1 \mathrm{~V} 4 \mathrm{~S}$ receptor grid by XP precision of the docking method. The docking glide score, binding free energy (using Prime MM-GBSA method), hydrogen bonding and $\pi-\pi$ interactions with the surrounding amino acids were studied to elucidate the binding affinities and appropriate alignment of all the ligand into the active site of 1V4S. The best-suited conformations of ligands, which were successful in reversing the protein in its original conformation and produced maximum dock score, were studied precisely.

\section{In-silico absorption, distribution, metabolism, excretion, and toxicity (ADMET) prediction}

Pharmacokinetic parameters (absorption, distribution, metabolism, and excretion) play a significant role in the discovery of novel drug candidates as many invented drugs fail in the development process. Hence, in-silico ADMET evaluation was computed using SwissADME [29] and PreADMET [30] web tools. The models used to predict the ADMET properties in this protocol are derived from a variety of experimental data sources and are cataloged in the product documentation.

\section{Results}

Chemistry

Ethyl [2-(2-methyl-4-oxoquinazolin-3(4H)-yl)-1,3-thiazol-4yl]acetate (3)

Yield: $70 \%$; m. p.: $140-142{ }^{\circ} \mathrm{C}$; $\mathrm{R}_{f}$ : 0.7; IR $(\mathrm{KBr}) \mathrm{cm}^{-1}$ : 2918 (Ali. C-H), $2850(\mathrm{C}-\mathrm{S}), 1691(\mathrm{C}=\mathrm{O}), 1587(\mathrm{C}=\mathrm{N})$, 1535 (Aro. $\mathrm{C}=\mathrm{C}), 1033(\mathrm{C}-\mathrm{O}) .{ }^{1} \mathrm{H}$ NMR $\left(\mathrm{CDCl}_{3}\right): \delta 1.3-$ $1.4\left(t, 3 H, J=6.8 \mathrm{~Hz}, \mathrm{CH}_{3}\right), 2.2\left(s, 3 \mathrm{H}, \mathrm{N}-\mathrm{C}-\mathrm{CH}_{3}\right), 3.8$ (s, $\left.2 \mathrm{H}, \mathrm{CH}_{2}-\mathrm{CO}\right), 4.25\left(q, 2 \mathrm{H}, J=4.2 \mathrm{~Hz}, \mathrm{O}-\mathrm{CH}_{2}\right), 6.8-$ $8.8(m, 5 H, A r-H) ;{ }^{13} \mathrm{C}$ NMR (DMSO- $\left.d_{6}\right): \delta 171.7$, $169.5,160.8,151.6,150.3,133.5,128.6,127.7,122.4$, 120.7, 104.4, 61.3, 38.6, 22.3, 14.1; HRMS (ESI) $\mathrm{m} / \mathrm{z}$ $(\mathrm{M}+1)$ 330; Anal. Calcd. for $\mathrm{C}_{16} \mathrm{H}_{15} \mathrm{~N}_{3} \mathrm{O}_{3} \mathrm{~S}$ : C, 58.34; $\mathrm{H}$, 4.59; N, 12.76; found: C, 58.28; H, 4.61; N, 12.78 .

2-[2-(2-Methyl-4-oxoquinazolin-3(4H)-yl)-1,3-thiazol-4-yl]-Nphenylacetamide (4)

Yield: 65\%; m. p.: $220-222^{\circ} \mathrm{C}$; IR $(\mathrm{KBr}) \mathrm{cm}^{-1}: 3421(\mathrm{~N}-$ H), 2933 (Ali. C-H), $1687(\mathrm{C}=\mathrm{O}), 1579(\mathrm{C}=\mathrm{N}), 1448(\mathrm{C}=$ C); ${ }^{1} \mathrm{H}$ NMR $\left(\mathrm{CDCl}_{3}\right): \delta 1.89\left(s, 3 H, \mathrm{CH}_{3}\right), 2.16(s, 2 \mathrm{H}$, $\left.\mathrm{CH}_{2}\right), 6.9-8.5$ (m, 10H, Ar-H), $13.8(s, 1 H, \mathrm{NH}) ;{ }^{13} \mathrm{C}$
NMR (DMSO- $\left.d_{6}\right): \delta 171.9,169.8,160.7,151.5,150.7$, $147.2,138.5,133.4,129.5,129.1,128.6,127.5,124.5$, 122.4, 121.6, 120.9, 104.5, 36.3, 22.2; HRMS (ESI) $\mathrm{m} / \mathrm{z}$ $(\mathrm{M}+1)$ 377; Anal. Calcd. for $\mathrm{C}_{20} \mathrm{H}_{16} \mathrm{~N}_{4} \mathrm{O}_{2} \mathrm{~S}$ : C, 63.81; H, 4.28; N, 14.88; found: C, 63.78; H, 4.31; N, 14.85 .

$\mathrm{N}$-(4-chlorophenyl)-2-[2-(2-methyl-4-oxoquinazolin-3(4H)-yl)1,3-thiazol-4-yl]acetamide (5)

Yield: 62\%; m. p.: $238-240{ }^{\circ} \mathrm{C}$; IR $(\mathrm{KBr}) \mathrm{cm}^{-1}: 3308(\mathrm{~N}-$ H), 2924 (Ali. C-H.), 1685 (C=O), 1585 (C=N.), 1301 $(\mathrm{C}=\mathrm{C}), 754(\mathrm{C}-\mathrm{Cl}) ;{ }^{1} \mathrm{H}$ NMR $\left(\mathrm{CDCl}_{3}\right): \delta 2.01(s, 3 H$, $\left.\mathrm{CH}_{3}\right), 2.26\left(s, 2 \mathrm{H}, \mathrm{CH}_{2}\right), 6.9-8.5(m, 9 H, \mathrm{Ar}-\mathrm{H}), 12.7$ (s, $1 H, \mathrm{NH}) ;{ }^{13} \mathrm{C}$ NMR (DMSO- $\left.d_{6}\right): \delta 171.8,169.9,160.8$, $151.7,150.9,147,137.6,134,133.6,129.4,129.2,128.7$, $127.5,122.3,121.7,121.5,120.8,104.5,36.3,22.5$; HRMS (ESI) $m / z(\mathrm{M}+1)$ 411; Anal. Calcd. for $\mathrm{C}_{20} \mathrm{H}_{15} \mathrm{ClN}_{4} \mathrm{O}_{2} \mathrm{~S}$ : C, 58.46; H, 3.68; N, 13.64; found: C, 58.48; H, 3.66; N, 13.66 .

$\mathrm{N}$-(4-fluorophenyl)-2-[2-(2-methyl-4-oxoquinazolin-3(4H)-yl)1,3-thiazol-4-yl]acetamide (6)

Yield: 67\%; m. p.: $235-238{ }^{\circ} \mathrm{C}$; IR $(\mathrm{KBr}) \mathrm{cm}^{-1}: 3213(\mathrm{~N}-$ H), 2926 (Ali. C-H), $1678(\mathrm{C}=\mathrm{O}), 1587(\mathrm{C}=\mathrm{N}), 1301(\mathrm{C}-$ F), $1242(\mathrm{C}=\mathrm{C}) ;{ }^{1} \mathrm{H}$ NMR $\left(\mathrm{CDCl}_{3}\right): \delta 1.93\left(s, 3 \mathrm{H}, \mathrm{CH}_{3}\right)$, $2.55\left(s, 2 \mathrm{H}, \mathrm{CH}_{2}\right), 6.8-8.2(m, 9 H, \mathrm{Ar}-\mathrm{H}), 13.1(s, 1 \mathrm{H}$, $\mathrm{NH}) ;{ }^{13} \mathrm{C}$ NMR (DMSO- $\left.d_{6}\right): \delta 171.9,169.8,160.8,158.6$, $151.8,150.9,147.2,134.2,133.6,128.9,127.5,123.4$, 122.4, 120.1, 115.6, 104.5, 36.3, 22.2; HRMS (ESI) $\mathrm{m} / \mathrm{z}$ $(\mathrm{M}+1)$ 395; Anal. Calcd. for $\mathrm{C}_{20} \mathrm{H}_{15} \mathrm{FN}_{4} \mathrm{O}_{2} \mathrm{~S}: \mathrm{C}, 60.90 ; \mathrm{H}$, 3.83; N, 14.20; found: C, 60.87; H, 3.85; N, 14.18 .

2-[2-(2-Methyl-4-oxoquinazolin-3(4H)-yl)-1,3-thiazol-4-yl]-N(3-methylphenyl) acetamide (7)

Yield: $75 \%$; m. p.: $218-220{ }^{\circ} \mathrm{C}$; IR $(\mathrm{KBr}) \mathrm{cm}^{-1}: 3210(\mathrm{~N}-$ H), 2919 (Ali. C-H), $1650(\mathrm{C}=\mathrm{O}), 1593(\mathrm{C}=\mathrm{N}), 1230(\mathrm{C}=$ C); ${ }^{1} \mathrm{H}$ NMR $\left(\mathrm{CDCl}_{3}\right): \delta 1.91\left(s, 3 H, \mathrm{CH}_{3}\right) .2 .1(s, 3 H$, $\left.\mathrm{Ar}-\mathrm{CH}_{3},\right), 3.2\left(s, 2 \mathrm{H}, \mathrm{CH}_{2}\right), 6.5-8.5(m, 9 H, \mathrm{Ar}-\mathrm{H}), 11.8$ $(s, 1 H, \mathrm{NH}) ;{ }^{13} \mathrm{C}$ NMR (DMSO- $\left.d_{6}\right): \delta 171.8,169.8,160.9$, $151.8,150.8,147.2,138.8,138.5,133.5,129.1,128.8$, $127.4,124.8,122.5,121.4,120.2,118.5,104.5,36.4,24.5$, 22.3; HRMS (ESI) $m / z(M+1)$ 391; Anal. Calcd. for $\mathrm{C}_{21} \mathrm{H}_{18} \mathrm{~N}_{4} \mathrm{O}_{2} \mathrm{~S}: \mathrm{C}, 64.60 ; \mathrm{H}, 4.65 ; \mathrm{N}, 14.35$; found: C, 64.56; H, 4.62; N, 14.36 .

2-[2-(2-Methyl-4-oxoquinazolin-3(4H)-yl)-1,3-thiazol-4-yl]-N(4-methylphenyl) acetamide (8)

Yield: 72\%; m. p.: $220-224{ }^{\circ} \mathrm{C}$; IR $(\mathrm{KBr}) \mathrm{cm}^{-1}: 3218(\mathrm{~N}-$ H), 2898 (Ali. C-H), $1652(\mathrm{C}=\mathrm{O}), 1596(\mathrm{C}=\mathrm{N}), 1228(\mathrm{C}=$ $\mathrm{C}) ;{ }^{1} \mathrm{H}$ NMR $\left(\mathrm{CDCl}_{3}\right): \delta 2.2\left(s, 3 \mathrm{H}, \mathrm{CH}_{3}\right), 2.4(s, 3 \mathrm{H}, \mathrm{Ar}-$ $\mathrm{H}), 2.7\left(s, 2 H, \mathrm{CH}_{2}\right), 6.7-8.5(m, 9 H, \mathrm{Ar}-\mathrm{H}), 12.1(s, 1 H$, $\mathrm{NH}) ;{ }^{13} \mathrm{C}$ NMR (DMSO- $\left.d_{6}\right): \delta 171.8,169.9,160.7,151.6$, $150.6,147.2,137.5,134.1,133.6,129.2,128.9,127.5$, $122.5,121.6,120.9,105.5,36.4,24.5,22.5$; HRMS (ESI) 
$m / z(\mathrm{M}+1)$ 391; Anal. Calcd. for $\mathrm{C}_{21} \mathrm{H}_{18} \mathrm{~N}_{4} \mathrm{O}_{2} \mathrm{~S}: \mathrm{C}$, 64.60; $\mathrm{H}, 4.65 ; \mathrm{N}, 14.35$; found: $\mathrm{C}, 64.62 ; \mathrm{H}, 4.64 ; \mathrm{N}, 14.39$.

$\mathrm{N}$-(3-methoxyphenyl)-2-[2-(2-methyl-4-oxoquinazolin-3(4H)yl)-1,3-thiazol-4-yl]acetamide (9)

Yield: 69\%; m. p.: $223-225{ }^{\circ} \mathrm{C}$; IR (KBr) cm ${ }^{-1}: 3220(\mathrm{~N}-$ H), 2890 (Ali. C-H), 1649 (C=O), $1586(\mathrm{C}=\mathrm{N}), 1280$ (C$\mathrm{O}), 1225(\mathrm{C}=\mathrm{C}) ;{ }^{1} \mathrm{H}$ NMR $\left(\mathrm{CDCl}_{3}\right): \delta 2.6\left(s, 2 \mathrm{H}, \mathrm{CH}_{2}\right)$, $2.8\left(s, 2 \mathrm{H}, \mathrm{CH}_{2}\right), 3.1\left(s, 3 \mathrm{H}, \mathrm{OCH}_{3}\right), 6.8-8.6(m, 9 H, \mathrm{Ar}-$ $\mathrm{H}), 12.3(s, 1 H, \mathrm{NH}) ;{ }^{13} \mathrm{C}$ NMR (DMSO- $\left.d_{6}\right): \delta 171.8$, $168.8,160.9,151.6,150.8,147.5,139.8,133.8,131,128.9$, 127.5, 122.4, 120.7, 113.7, 109.8, 104.9, 104.2, 55.9, 38.2, 22.6; HRMS (ESI) $m / z(M+1)$ 407; Anal. Calcd. for $\mathrm{C}_{21} \mathrm{H}_{18} \mathrm{~N}_{4} \mathrm{O}_{3} \mathrm{~S}$ : C, 62.05; $\mathrm{H}, 4.46 ; \mathrm{N}, 13.78$; found: $\mathrm{C}$, 62.12; H, 4.48; N, 13.76.

$\mathrm{N}$-(4-methoxyphenyl)-2-[2-(2-methyl-4-oxoquinazolin-3(4H)yl)-1,3-thiazol-4-yl]acetamide (10)

Yield: $71 \%$; m. p.: $225-227{ }^{\circ} \mathrm{C}$; IR (KBr) cm ${ }^{-1}: 3224(\mathrm{~N}-$ H), 2893 (Ali. C-H), 1648 (C=O), $1596(\mathrm{C}=\mathrm{N}), 1268$ (C$\mathrm{O}), 1228(\mathrm{C}=\mathrm{C}) ;{ }^{1} \mathrm{H}$ NMR $\left(\mathrm{CDCl}_{3}\right): \delta 2.5\left(s, 3 H, \mathrm{CH}_{3}\right)$, $2.8\left(s, 2 \mathrm{H}, \mathrm{CH}_{2}\right), 3.2\left(s, 3 \mathrm{H}, \mathrm{OCH}_{3}\right), 6.8-8.5(m, 9 H, \mathrm{Ar}-$ $\mathrm{H}), 11.8(s, 1 H, \mathrm{NH}) ;{ }^{13} \mathrm{C}$ NMR (DMSO- $\left.d_{6}\right): \delta 171.8$, $169.7,160.9,156.2,151.7,150.6,147.3,136.6,130.9$, $128.8,127.5,122.8,122.4,120.9,114.6,104.6,55.7,35.3$, 22.1; HRMS (ESI) $m / z(M+1)$ 407; Anal. Calcd. for $\mathrm{C}_{21} \mathrm{H}_{18} \mathrm{~N}_{4} \mathrm{O}_{3} \mathrm{~S}: \mathrm{C}, 62.05 ; \mathrm{H}, 4.46 ; \mathrm{N}, 13.78$; found: C, 62.09; H, 4.43; N, 13.79 .

\section{Ethyl [2-(2-ethyl-4-oxoquinazolin-3(4H)-yl)-1,3-thiazol-4- yl]acetate (12)}

Yield: $66 \%$; m. p.: $144-146{ }^{\circ} \mathrm{C}$; $\mathrm{R}_{f}: 0.7$; IR $(\mathrm{KBr}) \mathrm{cm}^{-1}$ : 2920(Ali. C-H), 2848 (C-S), 1689 (C=O), $1587(\mathrm{C}=\mathrm{N})$, 1532 (Aro. $\mathrm{C}=\mathrm{C}$ ), $1023(\mathrm{C}-\mathrm{O}) ;{ }^{1} \mathrm{H}$ NMR $\left(\mathrm{CDCl}_{3}\right): \delta 1.21$ $\left(t, 3 \mathrm{H}, J=3.4 \mathrm{~Hz}, \mathrm{CH}_{3}\right), 1.23\left(t, 3 \mathrm{H}, J=6.3 \mathrm{~Hz}, \mathrm{CH}_{3}\right)$, $2.69\left(q, 2 \mathrm{H}, \mathrm{J}=7.7 \mathrm{~Hz}, \mathrm{CH}_{2}\right), 3.6\left(s, 2 \mathrm{H}, \mathrm{CO}-\mathrm{CH}_{2}\right), 4.10$ $\left(q, 2 \mathrm{H}, \mathrm{J}=7.1 \mathrm{~Hz}, \mathrm{O}-\mathrm{CH}_{2}\right), 7.1-8.3(m, 5 \mathrm{H}, \mathrm{Ar}-\mathrm{H}) ;{ }^{13} \mathrm{C}$ NMR (DMSO- $\left.d_{6}\right): \delta 171.8,169.3,164,160.8,150.8$, 147.1, 133.5, 128.8, 127.4, 122.4, 120.7, 104.2, 61.5, 38.6, 24.1, 14.4, 7.1; HRMS (ESI) $m / z(M+1)$ 344; Anal. Calcd. for $\mathrm{C}_{17} \mathrm{H}_{17} \mathrm{~N}_{3} \mathrm{O}_{3} \mathrm{~S}$ : C, 59.46; $\mathrm{H}, 4.99 ; \mathrm{N}, 12.24$; found: $\mathrm{C}$, 59.44; H, 4.92; N, 12.36 .

\section{2-[2-(2-Ethyl-4-oxoquinazolin-3(4H)-yl)-1,3-thiazol-4-yl]-N- phenylacetamide (13)}

Yield: 67\%; m. p.: $218-220{ }^{\circ} \mathrm{C}$; IR (KBr) cm ${ }^{-1}: 3421$ (NH), 2933 (Ali. C-H), $1687(\mathrm{C}=\mathrm{O}), 1579(\mathrm{C}=\mathrm{N}), 1448(\mathrm{C}=$ C); ${ }^{1} \mathrm{H} \mathrm{NMR}\left(\mathrm{CDCl}_{3}\right): \delta 1.33\left(t, 3 \mathrm{H}, J=6.7 \mathrm{~Hz}, \mathrm{CH}_{3}\right)$, $2.16\left(q, 2 \mathrm{H}, \mathrm{J}=8.2 \mathrm{~Hz}, \mathrm{CH}_{2}\right), 2.86\left(s, 2 \mathrm{H}, \mathrm{CO}-\mathrm{CH}_{2}\right)$, 7.0-8.2 (m, 10H , Ar-H), 13.3 (s, 1H, NH); ${ }^{13} \mathrm{C}$ NMR $\left(\mathrm{DMSO}-d_{6}\right): \delta 171.7,169.9,165.2,160.7,150.7,147.3$, 138.6, 133.6, 129, 128.8, 127.5, 124, 122.2, 121.5, 120.7, 104.5, 36.2, 23.7; HRMS (ESI) $m / z(M+1)$ 391; Anal.
Calcd. for $\mathrm{C}_{21} \mathrm{H}_{18} \mathrm{~N}_{4} \mathrm{O}_{2} \mathrm{~S}: \mathrm{C}, 64.60 ; \mathrm{H}, 4.65 ; \mathrm{N}, 14.35$; found: $C, 64.59 ; \mathrm{H}, 4.63 ; \mathrm{N}, 14.32$.

\section{$\mathrm{N}$-(4-chlorophenyl)-2-[2-(2-ethyl-4-oxoquinazolin-3(4H)-yl)- 1,3-thiazol-4-yl]acetamide (14)}

Yield: 62\%; m. p.: $238-241{ }^{\circ} \mathrm{C}$; IR (KBr) cm ${ }^{-1}: 3308(\mathrm{~N}-$ H), 2924 (Ali. C-H), $1685(\mathrm{C}=\mathrm{O}), 1585(\mathrm{C}=\mathrm{N}), 1301(\mathrm{C}=$ C), $754(\mathrm{C}-\mathrm{Cl}) ;{ }^{1} \mathrm{H}$ NMR $\left(\mathrm{CDCl}_{3}\right): \delta 1.36(t, 3 H, J=6.8$ $\left.\mathrm{Hz}, \mathrm{CH}_{3}\right), 2.56\left(s, 2 \mathrm{H}, \mathrm{CO}-\mathrm{CH}_{2}\right), 2.28(q, 2 \mathrm{H}, J=6.5 \mathrm{~Hz}$, $\left.\mathrm{CH}_{2}\right)$, 6.9-8.5 (m, 9H, Ar-H), 13.1 (s, $\left.1 H, \mathrm{NH}\right) ;{ }^{13} \mathrm{C}$ NMR (DMSO- $\left.d_{6}\right): \delta 171.9,169.7,165,160.8,151,147.2$, 136.8, 133.6, 129.8, 129.1, 128.3, 127.5, 123.4,122.8, 120.6, 104.3, 36.5, 23.7, 5.6; HRMS (ESI) $m / z(M+1) 425$; Anal. Calcd. for $\mathrm{C}_{21} \mathrm{H}_{17} \mathrm{ClN}_{4} \mathrm{O}_{2} \mathrm{~S}$ : C, 59.36; $\mathrm{H}, 4.03 ; \mathrm{N}$, 13.19; found: C, 59.32; H, 4.06; N, 13.22.

\section{$\mathrm{N}$-(4-fluorophenyl)-2-[2-(2-ethyl-4-oxoquinazolin-3(4H)-yl)-} 1,3-thiazol-4-yl]acetamide (15)

Yield: 63\%; m. p.: $235-237{ }^{\circ} \mathrm{C}$; IR (KBr) cm ${ }^{-1}: 3213(\mathrm{~N}-$ H), 2926 (Ali. C-H), $1685(\mathrm{C}=\mathrm{O}), 1587(\mathrm{C}=\mathrm{N}), 1303(\mathrm{C}-$ F), $1242(\mathrm{C}=\mathrm{C}) ;{ }^{1} \mathrm{H}$ NMR $\left(\mathrm{CDCl}_{3}\right): \delta 1.17(t, 3 H, J=7.9$ $\left.\mathrm{Hz}, \mathrm{CH}_{3}\right), 2.04\left(q, 2 \mathrm{H}, J=6.4 \mathrm{~Hz}, \mathrm{CH}_{2}\right), 2.48(s, 2 \mathrm{H}$, $\left.\mathrm{CO}-\mathrm{CH}_{2}\right), 6.9-8.2(m, 9 H, \mathrm{Ar}-\mathrm{H}), 13.7(s, 1 H, \mathrm{NH}) ;{ }^{13} \mathrm{C}$ NMR (DMSO- $\left.d_{6}\right): \delta 171.6,168.7,162.3,161,158.7$, $150.1,147.5,136,133.2,128.9,127.9,123.6,122.1,120.9$, 115.2, 103.5, 36.4, 24.7, 6.7; HRMS (ESI) $m / z(M+1) 409$; Anal. Calcd. for $\mathrm{C}_{21} \mathrm{H}_{17} \mathrm{FN}_{4} \mathrm{O}_{2} \mathrm{~S}$ : C, 61.75; H, 4.20; N, 13.72; found: C, 61.69; H, 4.16; N, 13.76 .

2-[2-(2-Ethyl-4-oxoquinazolin-3(4H)-yl)-1,3-thiazol-4-yl]-N-(3methylphenyl)acetamide (16)

Yield: 72\%; m. p.: $240-243{ }^{\circ} \mathrm{C}$; IR (KBr) cm ${ }^{-1}: 3200(\mathrm{~N}-$ H), 2928 (Ali. C-H), $1678(\mathrm{C}=\mathrm{O}), 1577(\mathrm{C}=\mathrm{N}), 1240(\mathrm{C}=$ C); ${ }^{1} \mathrm{H} \mathrm{NMR}\left(\mathrm{CDCl}_{3}\right): \delta 1.09\left(t, 3 \mathrm{H}, J=5.8 \mathrm{~Hz}, \mathrm{CH}_{3}\right)$, $2.07\left(q, 2 \mathrm{H}, \mathrm{J}=7.6 \mathrm{~Hz}, \mathrm{CH}_{2}\right), 2.35\left(s, 3 \mathrm{H}, \mathrm{CH}_{3}\right), 3.12(s$, $\left.2 \mathrm{H}, \mathrm{CH}_{2}\right), 6.9-8.1(m, 9 H, \mathrm{Ar}-\mathrm{H}), 12.8(s, 1 H, \mathrm{NH}) ;{ }^{13} \mathrm{C}$ NMR (DMSO- $\left.d_{6}\right): \delta 170.5,169.8,159.7,158.1,150.5$, $145.6,139.1,138.5,133.4,128.9,128.2,127.9,124.8$, 122.9, 121.5, 120.2, 118.5, 103.7, 38.9, 23.7, 6.8; HRMS (ESI) $m / z(\mathrm{M}+1)$ 405; Anal. Calcd. for $\mathrm{C}_{22} \mathrm{H}_{20} \mathrm{~N}_{4} \mathrm{O}_{2} \mathrm{~S}$ : C, 65.33; H, 4.98; N, 13.85; found: C, 65.28; H, 4.92; N, 13.88 .

2-[2-(2-Ethyl-4-oxoquinazolin-3(4H)-yl)-1,3-thiazol-4-yl]-N-(4methylphenyl)acetamide (17)

Yield: 64\%; m. p.: $244-247{ }^{\circ} \mathrm{C}$; IR (KBr) cm ${ }^{-1}$ : $3243(\mathrm{~N}-$ H), 2948 (Ali. C-H), $1610(\mathrm{C}=\mathrm{O}), 1556(\mathrm{C}=\mathrm{N}), 1248(\mathrm{C}=$ C); ${ }^{1} \mathrm{H}$ NMR $\left(\mathrm{CDCl}_{3}\right): \delta 1.51\left(t, 3 \mathrm{H}, J=6.8 \mathrm{~Hz}, \mathrm{CH}_{3}\right), 2.1$ $\left(q, 2 H, J=7.4 \mathrm{~Hz}, \mathrm{CH}_{2}\right), 2.9\left(s, 3 H, \mathrm{CH}_{3}\right), 3.2(s, 2 \mathrm{H}$, $\left.\mathrm{CH}_{2}\right), 7.0-8.5(m, 9 H, \mathrm{Ar}-\mathrm{H}), 13.4(s, 1 H, \mathrm{NH}) ;{ }^{13} \mathrm{C}$ NMR (DMSO- $\left.d_{6}\right): \delta 172.9,168.8,166.7,161.7,150.8$, $148.9,137.9,136.2,133.7,129.9,128.3,127.2,122.8$, 122.2, 120.9, 106.2, 38.7, 26.2, 7.8; HRMS (ESI) $\mathrm{m} / z$ 
$(\mathrm{M}+1)$ 405; Anal. Calcd. for $\mathrm{C}_{22} \mathrm{H}_{20} \mathrm{~N}_{4} \mathrm{O}_{2} \mathrm{~S}$ : C, 65.33; $\mathrm{H}$, 4.98; N, 13.85; found: C, 65.36; H, 4.94; N, 13.81 .

\section{2-[2-(2-Ethyl-4-oxoquinazolin-3(4H)-yl)-1,3-thiazol-4-yl]-N-(3-} methoxyphenyl) acetamide (18)

Yield: 72\%; m. p.: $223-225{ }^{\circ} \mathrm{C}$; IR (KBr) cm ${ }^{-1}: 3312(\mathrm{~N}-$ H), 2912 (Ali. C-H), 1648 (C=O), $1532(\mathrm{C}=\mathrm{N}), 1275(\mathrm{C}=$ C); ${ }^{1} \mathrm{H} \mathrm{NMR}\left(\mathrm{CDCl}_{3}\right): \delta 1.05\left(t, 3 H, J=7.6 \mathrm{~Hz}, \mathrm{CH}_{3}\right), 2.2$ $\left(q, 2 \mathrm{H}, J=8.2 \mathrm{~Hz}, \mathrm{CH}_{2}\right), 3.1\left(s, 2 \mathrm{H}, \mathrm{CH}_{2}\right), 3.6(s, 3 \mathrm{H}$, $\left.\mathrm{CH}_{3}\right), 6.5-7.9(m, 9 H, \mathrm{Ar}-\mathrm{H}), 12.7(s, 1 H, \mathrm{NH}) ;{ }^{13} \mathrm{C}$ NMR (DMSO- $\left.d_{6}\right): \delta 171.4,167.9,163,161.4,151.7$, $148.9,141.2,136.4,131.5,129.8,127.2,121.9,120.7$, $112.9,110.9,105.6,103.8,56.3,38.2,26.3,8.8$; HRMS (ESI) $\mathrm{m} / z(\mathrm{M}+1) 421$; Anal. Calcd. for $\mathrm{C}_{22} \mathrm{H}_{20} \mathrm{~N}_{4} \mathrm{O}_{3} \mathrm{~S}$ : C, 62.84; H, 4.79; N, 13.32; found: C, 62.76; H, 4.82; N, 13.28 .

2-[2-(2-Ethyl-4-oxoquinazolin-3(4H)-yl)-1,3-thiazol-4-yl]-N-(4methoxyphenyl) acetamide (19)

Yield: 67\%; m. p.: $256-258{ }^{\circ} \mathrm{C}$; IR (KBr) cm ${ }^{-1}: 3342(\mathrm{~N}-$ H), 2967 (Ali. C-H), $1682(\mathrm{C}=\mathrm{O}), 1534(\mathrm{C}=\mathrm{N}), 1267(\mathrm{C}=$ C); ${ }^{1} \mathrm{H}$ NMR $\left(\mathrm{CDCl}_{3}\right): \delta 1.34\left(t, 3 \mathrm{H}, J=6.4 \mathrm{~Hz}, \mathrm{CH}_{3}\right)$, $2.43\left(q, 2 \mathrm{H}, J=7.7 \mathrm{~Hz}, \mathrm{CH}_{2}\right), 3.4\left(s, 2 \mathrm{H}, \mathrm{CH}_{2}\right), 3.9(s, 3 \mathrm{H}$, $\left.\mathrm{OCH}_{3}\right), 7.1-8.4(m, 9 H, \mathrm{Ar}-\mathrm{H}), 13.2(s, 1 H, \mathrm{NH}) ;{ }^{13} \mathrm{C}$ NMR (DMSO- $\left.d_{6}\right): \delta 169.8,168.7,159.1,158.5,157$, $151.2,148.3,134.5,131.6,129.8,128.4,122.9,122.4$, 120.2, 113.5, 104.7, 57.5, 38.8, 24.6, 7.8; HRMS (ESI) $\mathrm{m} / z$ $(\mathrm{M}+1)$ 421; Anal. Calcd. for $\mathrm{C}_{22} \mathrm{H}_{20} \mathrm{~N}_{4} \mathrm{O}_{3} \mathrm{~S}$ : C, 62.84; $\mathrm{H}$, 4.79; N, 13.32; found: C, 62.83; H, 4.76; N, 13.35 .

\section{Ethyl [2-(4-oxo-2-phenylquinazolin-3(4H)-yl)-1,3-thiazol-4- yl]acetate (21)}

Yield: $77 \%$; m. p.: $110-120{ }^{\circ} \mathrm{C}$; $\mathrm{R}_{f}$ : 0.7; IR (KBr) $\mathrm{cm}^{-1}$ : 3061 (Aro. C-H), 2613 (C-S), 1685 (C=O), 1587 (Aro. $\mathrm{C}=\mathrm{C}), 1580(\mathrm{C}=\mathrm{N}), 1234$ (Ali. $\mathrm{C}-\mathrm{O}) ;{ }^{1} \mathrm{H}$ NMR $\left(\mathrm{CDCl}_{3}\right)$ : $\delta 1.21\left(t, 3 \mathrm{H}, J=5.6 \mathrm{~Hz}, \mathrm{CH}_{3}\right), 3.8\left(s, 2 \mathrm{H}, \mathrm{CH}_{2}\right), 4.10(q$, $\left.2 \mathrm{H}, \mathrm{J}=6.8 \mathrm{~Hz}, \mathrm{O}-\mathrm{CH}_{2}\right), 7.2-8.5(m, 10 H, \mathrm{Ar}-\mathrm{H}) ;{ }^{13} \mathrm{C}$ NMR (DMSO- $\left.d_{6}\right): \delta 171.7,169.5,163,160.6,150.8$, 133.7, 130.1, 129.1, 128.8, 128.5, 128, 127.2, 122.5, 120.7, 104.4, 61.3, 38.6, 14.2; HRMS (ESI) $m / z(M+1)$ 392; Anal. Calcd. for $\mathrm{C}_{21} \mathrm{H}_{17} \mathrm{~N}_{3} \mathrm{O}_{3} \mathrm{~S}$ : C, 64.43; $\mathrm{H}, 4.38 ; \mathrm{N}$, 10.73; found: C, 64.44; H, 4.36; N, 10.75 .

2-[2-(4-Oxo-2-phenylquinazolin-3(4H)-yl)-1,3-thiazol-4-yl]-Nphenylacetamide (22)

Yield: 60\%; m. p.: $104-106{ }^{\circ} \mathrm{C}$; IR (KBr) cm ${ }^{-1}: 3367$ (N$\mathrm{H}), 3132$ (Aro. C-H), 1687 (C=O), $1535(\mathrm{C}=\mathrm{N}), 1450$ $(\mathrm{C}=\mathrm{C}) ;{ }^{1} \mathrm{H}$ NMR $\left(\mathrm{CDCl}_{3}\right): \delta 2.59\left(s, 2 \mathrm{H}, \mathrm{CH}_{2}\right), 7-8.9(m$, $15 H, \mathrm{Ar}-\mathrm{H}), 12.3(s, 1 H, \mathrm{NH}) ;{ }^{13} \mathrm{C}$ NMR (DMSO- $\left.d_{6}\right): \delta$ $172.3,169.7,166.1,161.2,152.2,150.6,138.5,134.7$, $131.2,130.9,130.1,129.5,128.2,127.4,125.7,124.2$, 122.4, 121.6, 120.2, 104.7, 38.2; HRMS (ESI) $m / z(M+1)$ 439; Anal. Calcd. for $\mathrm{C}_{25} \mathrm{H}_{18} \mathrm{~N}_{4} \mathrm{O}_{2} \mathrm{~S}: \mathrm{C}, 68.48 ; \mathrm{H}, 4.14 ; \mathrm{N}$, 12.78; found: C, 68.52; $\mathrm{H}, 4.16 ; \mathrm{N}, 12.74$.
$\mathrm{N}$-(4-chlorophenyl)-2-[2-(4-oxo-2-phenylquinazolin-3(4H)-yl)1,3-thiazol-4-yl]acetamide (23)

Yield: 70\%; m. p.: $112-114{ }^{\circ} \mathrm{C}$; IR (KBr) cm ${ }^{-1}: 3446(\mathrm{~N}-$ H), 3049 (Aro. C-H), 1685 (C=O), $1587(\mathrm{C}=\mathrm{N}), 1232$ $(\mathrm{C}=\mathrm{C}), 785(\mathrm{C}-\mathrm{Cl}) ;{ }^{1} \mathrm{H} \mathrm{NMR}\left(\mathrm{CDCl}_{3}\right): \delta 2.26(s, 2 \mathrm{H}$, $\left.\mathrm{CH}_{2}\right), 6.9-8.5(m, 14 H, \mathrm{Ar}-\mathrm{H}), 12.7(s, 1 H, \mathrm{NH}) ;{ }^{13} \mathrm{C}$ NMR (DMSO- $\left.d_{6}\right): \delta 171.6,169.7,163.6,161.4,160.5$, $152.5,151.2,137.5,133.8,130.6,129.9,129.5,129.1$, 128.6, 128.1, 127.8, 127.2, 126.3, 122.9, 122.6, 121, 106.4, 37.5; HRMS (ESI) $m / z(M+1)$ 473; Anal. Calcd. for $\mathrm{C}_{25} \mathrm{H}_{17} \mathrm{ClN}_{4} \mathrm{O}_{2} \mathrm{~S}$ : C, 63.49; $\mathrm{H}, 3.62 ; \mathrm{N}, 11.85$; found: $\mathrm{C}$, 63.44; H, 3.65; N, 11.81 .

\section{$\mathrm{N}$-(4-fluorophenyl)-2-[2-(4-oxo-2-phenylquinazolin-3(4H)-yl)-} 1,3-thiazol-4-yl]acetamide (24)

Yield: 68\%; m. p.: $130-140{ }^{\circ} \mathrm{C}$; IR (KBr) cm ${ }^{-1}: 3249(\mathrm{~N}-$ $\mathrm{H}), 2929$ (Aro. $\mathrm{C}-\mathrm{H}), 1680(\mathrm{C}=\mathrm{O}), 1587(\mathrm{C}=\mathrm{N}), 1342$ $(\mathrm{C}=\mathrm{C}), 1289(\mathrm{C}-\mathrm{F}) ;{ }^{1} \mathrm{H}$ NMR $\left(\mathrm{CDCl}_{3}\right): \delta 2.55(s, 2 \mathrm{H}$, $\left.\mathrm{CH}_{2}\right), 6.9-8.7(m, 14 H, \mathrm{Ar}-\mathrm{H}), 12.3(s, 1 H, \mathrm{NH}) ;{ }^{13} \mathrm{C}$ NMR (DMSO- $\left.d_{6}\right): \delta 172.6,169.7,163.7,161.5,158.2$, $152.7,135.5,133.2,130.6,129.8,129.2,128.5,128.1$, 127.2, 125.7, 123.8, 122.7, 120.8, 118.9, 106.8, 39.7; HRMS (ESI) $m / z \quad(\mathrm{M}+1) \quad 457$; Anal. Calcd. For $\mathrm{C}_{25} \mathrm{H}_{17} \mathrm{FN}_{4} \mathrm{O}_{2} \mathrm{~S}: \mathrm{C}, 65.78 ; \mathrm{H}, 3.75 ; \mathrm{N}, 12.27$; found: C, 65.81; H, 3.78; N, 12.25 .

\section{$\mathrm{N}$-(3-methylphenyl)-2-[2-(4-oxo-2-phenylquinazolin-3(4H)-} yl)-1,3-thiazol-4-yl]acetamide (25)

Yield: 66\%; m. p.: $118-121{ }^{\circ} \mathrm{C}$; IR (KBr) cm ${ }^{-1}: 3210(\mathrm{~N}-$ H), 3087 (Aro. C-H), 2897 (Ali. C-H), 1650 (C=O), $1598(\mathrm{C}=\mathrm{N}), 1280(\mathrm{C}=\mathrm{C}) ;{ }^{1} \mathrm{H} \operatorname{NMR}\left(\mathrm{CDCl}_{3}\right): \delta 2.29(s$, $\left.3 \mathrm{H}, \mathrm{CH}_{3}\right), 2.7\left(s, 2 \mathrm{H}, \mathrm{CH}_{2}\right), 6.5-8.4(m, 14 H, \mathrm{Ar}-\mathrm{H})$, $11.6(s, 1 H, \mathrm{NH}) ;{ }^{13} \mathrm{C}$ NMR (DMSO- $\left.d_{6}\right): \delta 171.8,169.5$, $163.2,161.6,152.4,150.6,139.8,138.2,133.9,130.6$, $129.9,129.6,128.6,128.2,127.9,126.7,126.2,124.1$, 122.7, 121.7, 120.9, 118.8, 105.2, 38.6, 26.2; HRMS (ESI) $m / z(\mathrm{M}+1)$ 453; Anal. Calcd. for $\mathrm{C}_{26} \mathrm{H}_{20} \mathrm{~N}_{4} \mathrm{O}_{2} \mathrm{~S}$ : C, 69.01; $\mathrm{H}, 4.45$; N, 12.38; found: C, 69.06; H, 4.48; N, 12.43 .

\section{$\mathrm{N}$-(4-methylphenyl)-2-[2-(4-oxo-2-phenylquinazolin-3(4H)-} yl)-1,3-thiazol-4-yl]acetamide (26)

Yield: 73\%; m. p.: $124-126{ }^{\circ} \mathrm{C}$; IR (KBr) cm ${ }^{-1}$ : $3220(\mathrm{~N}-$ H), 3027 (Aro. C-H), 2876 (Ali. C-H), $1652(\mathrm{C}=\mathrm{O})$, 1594 $(\mathrm{C}=\mathrm{N}), 1238(\mathrm{C}=\mathrm{C}) ;{ }^{1} \mathrm{H}$ NMR $\left(\mathrm{CDCl}_{3}\right): \delta 2.7(s, 3 H$, $\left.\mathrm{CH}_{3}\right), 3.0\left(s, 2 \mathrm{H}, \mathrm{CH}_{2}\right), 7-8.5(m, 14 H, \mathrm{Ar}-\mathrm{H}), 12.1(s$, $1 H, \mathrm{NH}) ;{ }^{13} \mathrm{C}$ NMR (DMSO- $\left.d_{6}\right): \delta 170.6,168.9,162$, $161.7,154.9,152.3,136.3,135.6,134.9,130.4,129.4$, $128.8,128.5,128.1,127.2,125.5,122.6,121.8,120.5$, 105.6, 38.4, 28.6; HRMS (ESI) $m / z(M+1)$ 453; Anal. Calcd. for $\mathrm{C}_{26} \mathrm{H}_{20} \mathrm{~N}_{4} \mathrm{O}_{2} \mathrm{~S}$ : C, 69.01; H, 4.45; N, 12.38; found: C, 69.09; H, 4.39; N, 12.41 . 
$\mathrm{N}$-(3-methoxylphenyl)-2-[2-(4-oxo-2-phenylquinazolin-3(4H)yl)-1,3-thiazol-4-yl]acetamide (27)

Yield: 63\%; m. p.: $146-148{ }^{\circ} \mathrm{C}$; IR $(\mathrm{KBr}) \mathrm{cm}^{-1}: 3220$ $(\mathrm{N}-\mathrm{H}), 3031$ (Aro. $\mathrm{C}-\mathrm{H}), 2890$ (Ali. $\mathrm{C}-\mathrm{H}), 1652(\mathrm{C}=$ O), $1586(\mathrm{C}=\mathrm{N}), 1225(\mathrm{C}=\mathrm{C}) ;{ }^{1} \mathrm{H}$ NMR $\left(\mathrm{CDCl}_{3}\right): \delta 2.7$ $\left(s, 3 H, \mathrm{CH}_{3}\right), 3.3\left(s, 2 H, \mathrm{CH}_{2}\right), 6.7-8.2(m, 14 H, \mathrm{Ar}-$ $\mathrm{H}), 12.1(s, 1 H, \mathrm{NH}) ;{ }^{13} \mathrm{C}$ NMR (DMSO- $\left.d_{6}\right): \delta 172.6$, $168.4,163.9,160.5,154.3,152.2,139.7,133.5,131.2$, $130.5,129.4,129.2,128.8,128.2,127.2,126.8,122.6$, 120.3, 113.6, 109.2, 104.8, 103.2, 56.7, 40.2; HRMS (ESI) $m / z(\mathrm{M}+1)$ 470; Anal. Calcd. for $\mathrm{C}_{26} \mathrm{H}_{20} \mathrm{~N}_{4} \mathrm{O}_{2} \mathrm{~S}$ : C, 66.65; H, 4.30; N, 11.96; found: $\mathrm{C}, 66.61 ; \mathrm{H}, 4.34$; $\mathrm{N}, 11.91$.

$\mathrm{N}$-(4-methoxylphenyl)-2-[2-(4-oxo-2-phenylquinazolin-3(4H)yl)-1,3-thiazol-4-yl]acetamide (28)

Yield: $74 \%$; m. p.: $151-153{ }^{\circ} \mathrm{C}$; IR (KBr) cm ${ }^{-1}: 3218$ $(\mathrm{N}-\mathrm{H}), 3029$ (Aro. $\mathrm{C}-\mathrm{H}), 2898$ (Ali. $\mathrm{C}-\mathrm{H}), 1648(\mathrm{C}=$ O), $1596(\mathrm{C}=\mathrm{N}), 1266(\mathrm{C}=\mathrm{C}) ;{ }^{1} \mathrm{H}$ NMR $\left(\mathrm{CDCl}_{3}\right): \delta 2.6$ $\left(s, 3 H, \mathrm{CH}_{3}\right), 3.1\left(s, 2 H, \mathrm{CH}_{2}\right), 6.8-8.5(m, 14 H$, Ar$\mathrm{H}), 11.8(s, 1 H, \mathrm{NH}) ;{ }^{13} \mathrm{C}$ NMR (DMSO- $\left.d_{6}\right): \delta 171.1$, $168.2,164.2,160.6,156.4,153.7,151.2,134.8,130.3$, $129.7,129.2,128.8,128.5,127.8,127.2,125,124$, 122.7, 121.3, 120.2, 116.4, 105.6, 56.8, 42.6; HRMS (ESI) $\mathrm{m} / z(\mathrm{M}+1) 469$; Anal. Calcd. for $\mathrm{C}_{26} \mathrm{H}_{20} \mathrm{~N}_{4} \mathrm{O}_{2} \mathrm{~S}$ : C, 66.65; H, 4.30; N, 11.96; found: C, 66.63; H, 4.37; $\mathrm{N}, 11.93$.

\section{Pharmacological studies Acute toxicity studies}

The toxicity of selected compounds $(3,4,6,12,13,21$, and 27) was assessed by subjecting them to acute toxicity study in rats according to OECD guidelines. The toxicity study revealed that in each group of tested compounds, three or more animals survived of the five animals which were administered with $2000 \mathrm{mg} / \mathrm{kg}$ oral dose. Also, none of the five animals in the groups had shown any toxic effects during the observation period of 14 days.

\section{In vitro GK assay}

The $\mathrm{EC}_{50}$ values (Table 1 ) obtained by conducting human GK activation assay for all the newly synthesized compounds (3, 4-10, 12, 13-19, 21, 22-28) showed less ability of these compounds to stimulate GK for the conversion of glucose to glucose-6-phosphate, when compared with activating properties of established standard such as RO-28-1675, piragliatin. The EC 50 values of compounds $3, \mathbf{4}, \mathbf{5}, \mathbf{6}$, and 12 are prominent and encouraging.

\section{Oral glucose tolerance test (OGTT)}

The selected compounds $(3,4,5,6,12,13,21$, and 22) screened by the in vitro studies and substitution pattern
Table 1 In vitro Glucokinase assay and glide score of synthesized compounds (3, 4-10, 12, 13-19, 21, 22-28)

\begin{tabular}{|c|c|c|c|c|c|}
\hline \multirow[t]{2}{*}{ Compounds } & \multicolumn{4}{|c|}{ Human GK Activation, $\mathrm{EC}_{50}(\mathrm{nM})$} & \multirow{2}{*}{$\begin{array}{l}\text { Glide } \\
\text { score }\end{array}$} \\
\hline & 1 & $\|$ & III & Mean & \\
\hline 3 & 566 & 647 & 685 & 632 & -6.44 \\
\hline 4 & 580 & 450 & 520 & 516 & -6.24 \\
\hline 5 & 944 & 832 & 846 & 874 & -4.89 \\
\hline 6 & 780 & 890 & 830 & 833 & -4.95 \\
\hline 7 & 1550 & 1420 & 1480 & 1483 & -3.64 \\
\hline 8 & 1330 & 1250 & 1280 & 1286 & -3.85 \\
\hline 9 & 1190 & 1310 & 1215 & 1238 & -4.29 \\
\hline 10 & 1240 & 1290 & 1355 & 1295 & -4.56 \\
\hline 12 & 1020 & 939 & 878 & 945 & -5.37 \\
\hline 13 & 1055 & 1360 & 1195 & 1203 & -5.15 \\
\hline 14 & 954 & 1096 & 1033 & 1027 & -5.55 \\
\hline 15 & 1021 & 1040 & 1166 & 1075 & -4.96 \\
\hline 16 & 1440 & 1720 & 1585 & 1581 & -3.33 \\
\hline 17 & 1510 & 1230 & 1390 & 1376 & -3.42 \\
\hline 18 & 1110 & 1416 & 1340 & 1288 & -3.66 \\
\hline 19 & 1070 & 1260 & 1290 & 1206 & -3.75 \\
\hline 21 & 1240 & 1355 & 1180 & 1258 & -4.12 \\
\hline 22 & 1480 & 1550 & 1583 & 1537 & -3.96 \\
\hline 23 & 1610 & 1840 & 1530 & 1660 & -3.54 \\
\hline 24 & 1080 & 1350 & 1205 & 1211 & -3.62 \\
\hline 25 & 1680 & 1845 & 1620 & 1715 & -2.94 \\
\hline 26 & 1870 & 1560 & 1480 & 1636 & -3.02 \\
\hline 27 & 1755 & 1485 & 1950 & 1730 & -3.32 \\
\hline 28 & 1355 & 1520 & 1450 & 1441 & -3.37 \\
\hline RO-28-1675 & 353 & 388 & 405 & 382 & -6.39 \\
\hline Piragliatin & 365 & 395 & 420 & 393 & -7.89 \\
\hline
\end{tabular}

were further evaluated for their glucose-lowering effect in animal models by OGTT assay. The tested compounds showed a significant reduction in blood glucose levels, however, with a degree of variation, when compared with control in $0-120 \mathrm{~min}$ at a dose of $20 \mathrm{mg} / \mathrm{kg}$ (Fig. 2).

\section{In silico docking studies}

Molecular docking study was executed to understand the probable binding interactions of compounds (ligands) into the active site of the $1 \mathrm{~V} 4 \mathrm{~S}$ receptor. The glide score for all the newly synthesized compounds $(3-10,12-19,21-28)$ are reported in Table 1 along with a pharmacological activity to correlate the data. The binding free energy, hydrogen bonding, and $\pi-\pi$ interaction with the active site of $1 \mathrm{~V} 4 \mathrm{~S}$ of most active compounds 3 and 4 having highest glide scores are illustrated in Table 2 along with 


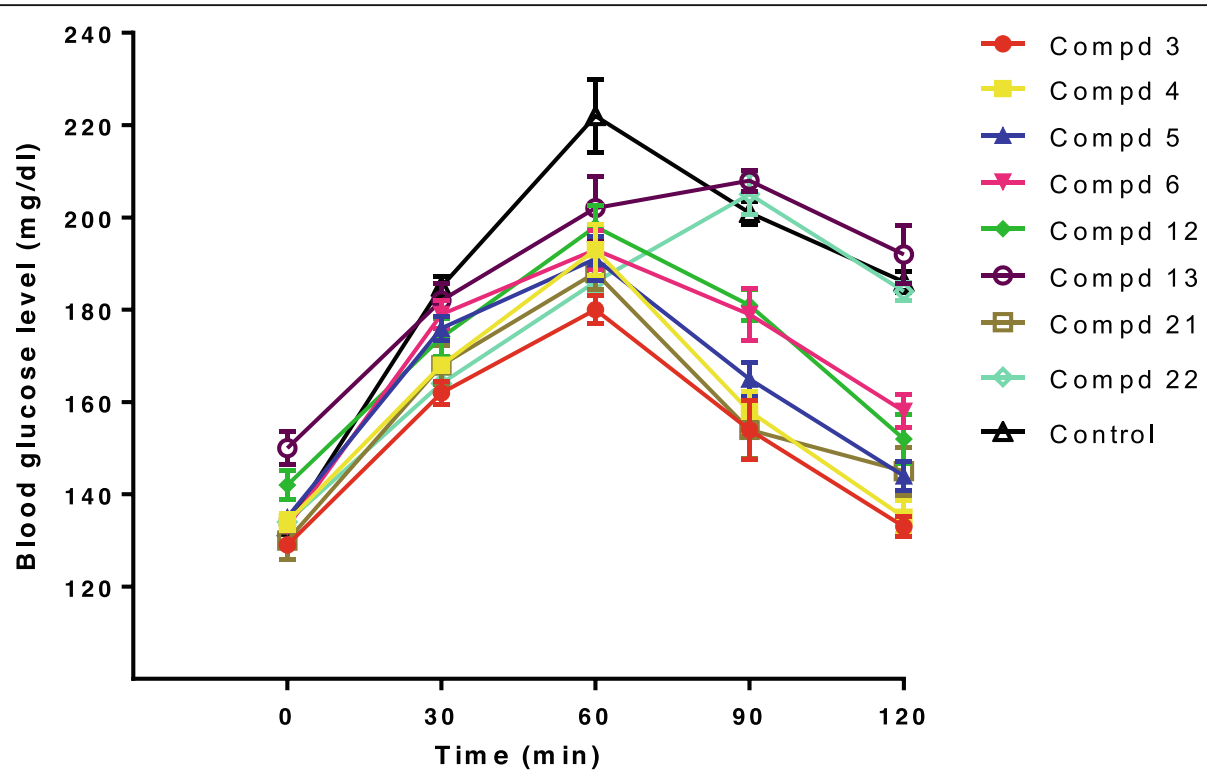

Fig. 2 In vivo OGTT of the selected compounds in normal rats at different time intervals. Data were analyzed by one-way ANOVA and expressed as mean $\pm \operatorname{SEM}(n=6), p<0.05$

standards RO-28-1675 and piragliatin. The glide score of the compounds was found to be in the range of -2.94 to - 6.44. Figs. 3 and 4 illustrate the binding poses as well as interactions of the compounds 3 and 4 having the highest glide score. The most active compounds 3 and 4 in the series in terms of GK activation and glide score are studied and discussed precisely.

\section{In silico ADMET studies}

ADMET prediction of the selected compounds $(\mathbf{3}, \mathbf{4}, \mathbf{5}$, $6,12,13,21$, and 22) were carried out and parameters like hydrogen bond acceptor, hydrogen bond donor, number of rotatable bonds, topological polar surface area, partition coefficient, aqueous solubility, bloodbrain barrier permeability, cytochrome-P2D6 inhibitor

Table 2 Binding free energy, hydrogen bonding, and $\pi-\pi$ interactions of most active quinazolinone derivatives (compounds $\mathbf{3}$ and 4) with key amino acid residues of the $1 \mathrm{~V} 4 \mathrm{~S}$ receptor

\begin{tabular}{|c|c|c|c|c|c|c|}
\hline \multirow[t]{2}{*}{ Compound } & \multirow[b]{2}{*}{$\begin{array}{l}\text { Binding } \\
\text { free } \\
\text { energy } \\
\text { MMGB/ } \\
\text { SA } \\
\text { (kcal/ } \\
\text { mol) }\end{array}$} & \multicolumn{3}{|c|}{ Hydrogen bonding } & \multicolumn{2}{|l|}{$\pi-\pi$ interaction } \\
\hline & & Atom of ligand & Amino acids & Distance $(\AA)$ & Ring of ligand & Amino acid \\
\hline \multirow[t]{2}{*}{3} & \multirow[t]{2}{*}{-53.51} & O & ARG 63 & 1.82 & \multirow[t]{2}{*}{ Quinazolinone } & \multirow[t]{2}{*}{ Tyr214 } \\
\hline & & $\mathrm{N}$ & THR 65 & 1.91 & & \\
\hline \multirow[t]{5}{*}{4} & \multirow[t]{5}{*}{-46.26} & $\mathrm{H}$ & TYR 61 & 2.01 & \multirow[t]{5}{*}{ Nil } & \multirow[t]{5}{*}{ Nil } \\
\hline & & $\mathrm{N}$ & ARG 63 & 2.59 & & \\
\hline & & $\mathrm{N}$ & TYR 214 & 2.14 & & \\
\hline & & H (Arom.) & TYR 61 & 2.60 & & \\
\hline & & $\mathrm{H}$ (Arom.) & ASP 158 & 2.52 & & \\
\hline \multirow[t]{4}{*}{ RO-28-1675 } & \multirow[t]{4}{*}{-60.53} & $\mathrm{O}$ & THR 65 & 1.70 & \multirow[t]{4}{*}{ Thiazole } & \multirow[t]{4}{*}{ Tyr 214} \\
\hline & & $\mathrm{H}$ (Arom.) & ARG 63 & 2.45 & & \\
\hline & & H (Arom.) & ARG 63 & 2.43 & & \\
\hline & & H (Arom.) & CYS 220 & 2.64 & & \\
\hline \multirow[t]{3}{*}{ Piragliatin } & \multirow[t]{3}{*}{-63.48} & $\mathrm{~N}$ & ARG 63 & 2.04 & \multirow[t]{3}{*}{ Nil } & \multirow[t]{3}{*}{ Nil } \\
\hline & & $\mathrm{H}$ & ARG 63 & 2.22 & & \\
\hline & & $\mathrm{O}$ & GLN 98 & 2.05 & & \\
\hline
\end{tabular}



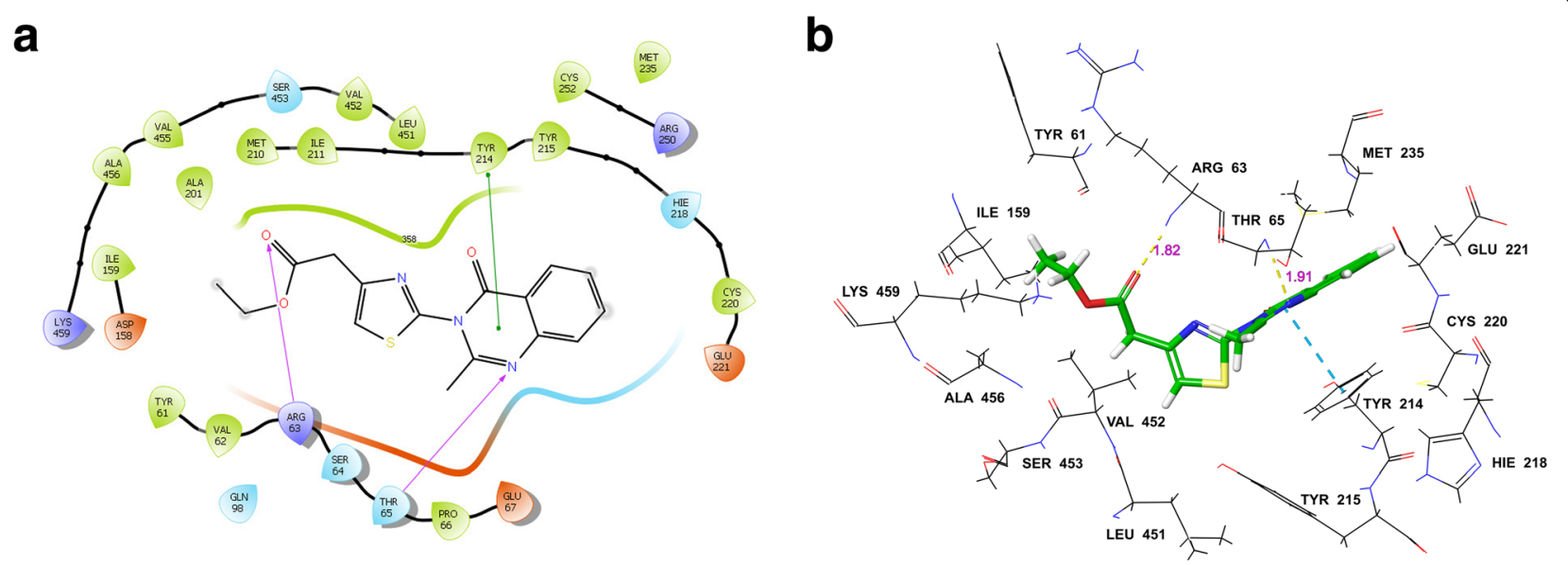

Fig. $32 \mathrm{D}$ and 3D docking pose displaying interaction of compound 3 in the binding site of $1 \mathrm{~V} 4 \mathrm{~S}$. a Hydrogen bonds are shown by pink lines, and the arrow points to the hydrogen bond acceptor, $\pi-\pi$ interactions by a solid green line and hydrophobic interactions by green amino acid residues; $\mathbf{b}$ Hydrogen bonds with yellow dot lines visualized as interaction of functional groups with an amino acid, blue dot lines indicate hydrogen bonding with aromatic hydrogen

(CYP2D6), Lipinski-no. of violations, hERG inhibition, carcinogenicity, and human intestinal absorption were studied and reported in Table 3. All the compounds tested were found to be non-toxic and ensured lead likeliness.

\section{Discussion}

\section{Chemistry}

The present study describes the synthesis of 2substituted quinazolinone thiazole acetates $(3,12,21)$ and 2-substituted quinazolinone thiazole acetamides [(4-10), (13-19), (22-28)]. The compounds were synthesized in satisfactory yield $(60-75 \%)$ as illustrated in Scheme 1, and physical properties and spectral data characterized their structures. The compounds were purified by recrystallization and column chromatography. Single spot TLC reports confirm the purity and homogeneity of synthesized compounds. The IR spectrum of compounds 2-substituted quinazolinone thiazole acetate $(3,12$, and 21$)$ showed the $\mathrm{C}=\mathrm{O}$ stretching in the range $1691-1685 \mathrm{~cm}^{-1}$ whereas, 2 -substituted quinazolinone thiazole acetamide (4-10), (13-19), and (22-28) revealed the presence of amide carbonyl (CO$\mathrm{NH})$ in the region of $1687-1610 \mathrm{~cm}^{-1}$ and amide $\mathrm{N}-\mathrm{H}$ in the region of $3446-3200 \mathrm{~cm}^{-1} .{ }^{1} \mathrm{H}$ NMR spectra of 2substituted quinazolinone thiazole acetate $(3,12$, and 21$)$ showed the signals for $-\mathrm{CH}_{2} \mathrm{CH}_{3}$ in the range of $\delta 1.21-$ 4.25 and aromatic protons at $\delta 6.8-8.8$. Further, 2-
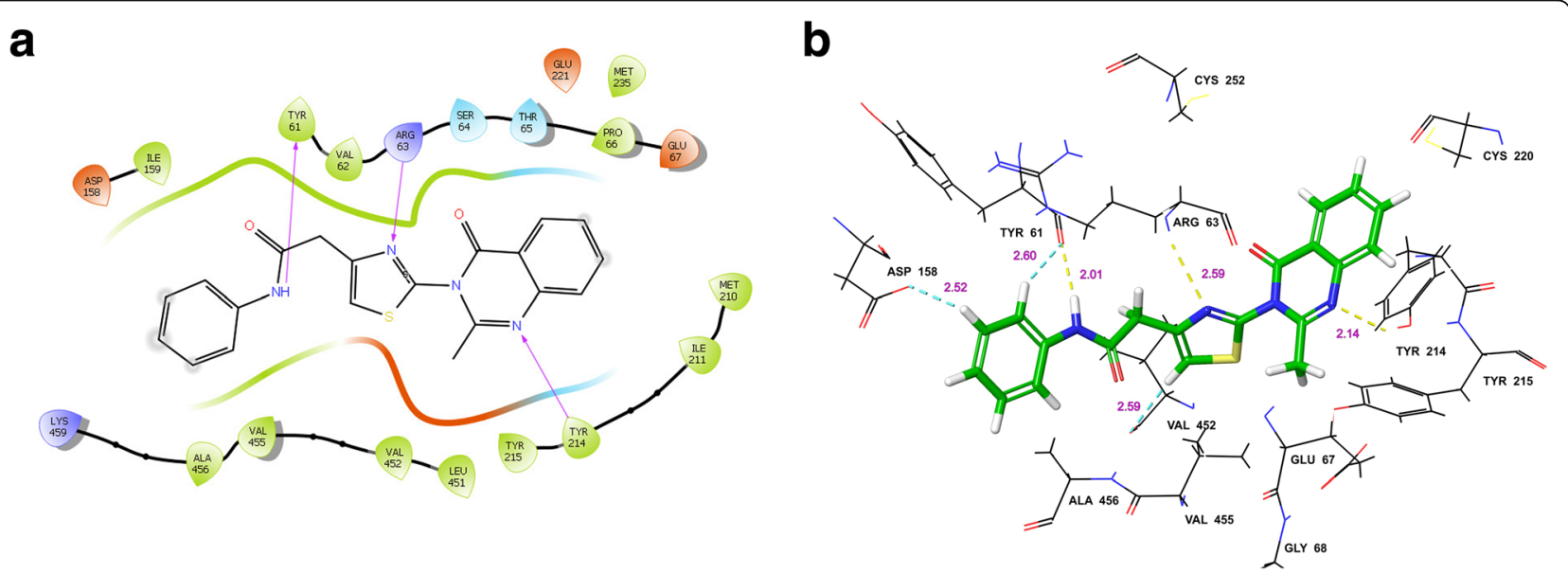

Fig. 4. $2 \mathrm{D}$ and $3 \mathrm{D}$ docking pose displaying interaction of compound 4 in the binding site of $1 \mathrm{~V} 4 \mathrm{~S}$. (a) Hydrogen bonds are shown by pink lines, and the arrow points to the hydrogen bond acceptor, $\pi-\pi$ interactions by a solid green line and hydrophobic interactions by green amino acid residues; (b) Hydrogen bonds with yellow dot lines visualized as interaction of functional groups with an amino acid, blue dot lines indicate hydrogen bonding with aromatic hydrogen. 
Table 3 In-Silico ADMET predictions using SwissADME and PreADMET software

\begin{tabular}{|c|c|c|c|c|c|c|c|c|c|c|c|c|}
\hline Compound no. & $\mathrm{HBA}$ & $\mathrm{HBD}$ & n-rotb & TPSA & $\begin{array}{l}\text { Log } \\
P_{\mathrm{o} / \mathrm{w}}\end{array}$ & $\log S$ & BBB & CYP2D6 inhibitor & Lipinski & hERG_inhibition & $\begin{array}{l}\text { Carcino } \\
\text { test }\end{array}$ & $\begin{array}{l}\text { HIA } \\
(\%)\end{array}$ \\
\hline 3 & 5 & 5 & 5 & 102.32 & 2.6 & -3.76 & No & No & 0 & ++ & negative & 98.64 \\
\hline 4 & 4 & 1 & 5 & 105.12 & 3.14 & -4.46 & No & No & 0 & + & negative & 97.45 \\
\hline 5 & 4 & 1 & 5 & 105.12 & 3.71 & -5.11 & No & No & 0 & ++ & negative & 96.95 \\
\hline 6 & 5 & 1 & 5 & 105.12 & 3.49 & -4.56 & No & No & 0 & ++ & positive & 97.44 \\
\hline 12 & 5 & 0 & 6 & 102.32 & 2.86 & -4.25 & No & No & 0 & ++ & negative & 98.94 \\
\hline 13 & 5 & 1 & 6 & 105.12 & 3.76 & -5.06 & No & No & 0 & ++ & positive & 97.44 \\
\hline 21 & 5 & 0 & 6 & 102.32 & 3.63 & -5.49 & No & No & 0 & + & negative & 99.56 \\
\hline$=22$ & 4 & 1 & 6 & 105.12 & 4.15 & -6.18 & No & No & 0 & + & negative & 96.86 \\
\hline
\end{tabular}

$H B A$ hydrogen bond acceptor, $\leq 10 ; H B D$ hydrogen bond donor, $\leq 5 ; n$-rotb no. of rotatable bonds, $\leq 10 ;$ TPSA topological polar surface area, $\leq 130 \AA^{2} ;$ Log Po/w octanol/water partition coefficient, -0.7 to +5.0 ; Log $S$, aqueous solubility scale: Insoluble $<-10<$ Poorly $<-6<$ Moderately $<-4<$ Soluble $<-2<$ Very Soluble $<0<$ Highly soluble; BBB blood-brain barrier permeability, central nervous system toxicity; CYP2D6 inhibitor hepatotoxicity; Lipinski number of violations of Lipinski's rule of five, maximum is 4 ; $h E R G$ inhibition high risk: +++ , medium risk: ++ , low risk: + ; carcino test carcinogenicity, positive or negative; HIA human intestinal absorption.

substituted quinazolinone thiazole acetamide [(4-10), (13-19), and (22-28)] exhibits one singlet for acetamide proton at $\delta 11.6-13.8$ which indicate the formation of these compounds. The triplet signal at around $\delta 1.05-$ 1.51 for $\underline{\mathrm{CH}}_{2}$ and quartet signal at $\delta 2.04-2.56$ for $\underline{\mathrm{CH}}_{3}$ of an ethyl group at position-2 of quinazolinone ring for compounds 13-19 was observed. However, all the other protons were resolved in appropriate regions confirming the assigned structures. The ${ }^{13} \mathrm{C}$ NMR signals at around $\delta 134.5-139.8$ for the carbon of $\mathrm{C}_{6} \mathrm{H}_{5}-\mathrm{NH}$ and characteristic signals of the $-\mathrm{C}=\mathrm{O}$ carbonyl group at $\delta 167.9-$ 169.9 were observed thus confirming the formation of an amide bond. Mass spectrum as $\mathrm{M}+1$ shows the molecular ion peaks of the synthesized quinazolinone. Elemental analysis data were found in the range of \pm $0.4 \%$ for the theoretical values of the analyzed elements (C, H, N).

\section{Pharmacological evaluation}

In line with OECD guideline, $\mathrm{LD}_{50}$ of the tested compounds was considered higher than test dose $(2000 \mathrm{mg} /$ $\mathrm{kg}$ ), and due to lack of observable toxicity at $2000 \mathrm{mg} / \mathrm{kg}$ dose, no determination of actual $\mathrm{LD}_{50}$ was carried out.

In vitro and in vivo models were used for the evaluation of glucose-lowering and glucokinase activation property. Human GK activation assay and oral glucose tolerance test (OGTT) are employed widely for the determination of the said activity. The most potent, amongst the tested compounds in GK activation assay was compound 4; having 2-methyl and phenylamido residues in its structure. The unsubstituted methyl-oxoquinazolinthiazol-phenylacetamide derivative (compound 4) demonstrated maximum $\mathrm{GK}$ activation $\left(\mathrm{EC}_{50}=516 \mathrm{nM}\right)$. The methyl-oxoquinazolin-thiazole acetate (compound 3) showed GK activation of $\mathrm{EC}_{50}=632 \mathrm{nM}$. The chloro(compound 5) and fluoro- (compound 6) substituted methyl-oxoquinazolin-thiazol-phenylacetamide derivatives and ethyl-oxoquinazolin-thiazole acetate (compound 12) exhibited good $\mathrm{GK}$ activation potency $\left(\mathrm{EC}_{50}=874 \mathrm{nM}\right.$, $\mathrm{EC}_{50}=833 \mathrm{nM}$, and $\mathrm{EC}_{50}=945 \mathrm{nM}$, respectively) while remaining compounds showed $\mathrm{EC}_{50}>1000 \mathrm{nM}$. Also, exploring the human GK activation data, it can be observed that as the chain elongates from methyl, ethyl to phenyl at 2-position of oxoquinazoline, the GK activity diminishes.

In the OGTT assay, the compounds 3 and 4 showed prominent activity and decreased the blood glucose level almost to normal at $120 \mathrm{~min}(133$ and $135 \mathrm{mg} / \mathrm{dL}$, respectively). The compounds $5,6,12$, and 21 demonstrated a moderate glucose-lowering effect $(144,158$, 152 , and $145 \mathrm{mg} / \mathrm{dL}$, respectively), whereas the compounds 13 and 22 were found to be ineffective on comparing with control. The data were statistically analyzed by one way ANOVA and expressed as mean \pm SEM $(n=$ $6, p<0.05)$. The results of OGTT were in concordance with the results of human GK activation assay. When results of GK activation assay and OGTT studied together, it was observed that compounds 4, 13, and 22 possessing non-substituted phenylamido residues shown prominent activity than compounds with halogen, methyl or methoxy substituted phenylamido groups. Moreover, compounds 3-10 with 2-methyl substitution on quinazolinone nucleus instigated much better activity than compounds 12-19 with 2-ethyl substitution and compounds $21-28$ with 2phenyl substitution.

\section{Molecular docking studies}

The compound 3 showed binding energy - $53.51 \mathrm{kcal} /$ mol and considerably high glide score -6.44 , slightly greater than one of the standard RO-28-1675 (glide score - 6.39). It binds with the active site of $1 \mathrm{~V} 4 \mathrm{~S}$ receptor forming a two-hydrogen bond between carbonyl oxygen $\mathrm{C}=\mathrm{O}$ of acetate group and Arg63 at a distance of $1.82 \AA$ and nitrogen $\mathrm{C}=\mathrm{N}$ of quinazolinone ring and Thr65 at a distance of $1.91 \AA$ which anchors ligand in 
the allosteric site of the enzyme. Additionally, the quinazolinone ring was found to have $\pi-\pi$ interaction with Tyr214. Moreover, the compound showed hydrophobic interactions with Met235, Cys252, Tyr215, Leu451, Val452, Ile211, Met210, Val455, Ala201, Ala456, Ile159, Tyr61, Val62, Pro66, and Cys220 (Fig. 3).

Compound 4, the most active compound in the series, showed the binding energy of -46.26 and glide score of - 6.24 which is comparatively less than compound 3 and standards. The compound 4 showed three hydrogen bond interactions; one between $\mathrm{NH}$ of the amide group and Tyr61 at a distance of $2.01 \AA$; second between $\mathrm{N}$ of thiazole ring and Arg63 at a distance of 2.59, and third between nitrogen $\mathrm{C}=\mathrm{N}$ of quinazolinone ring and Tyr214 at a distance of $2.14 \AA$. Additional hydrogen bonds are also observed in 3D docking pose between hydrogens of substituted aromatic ring and Tyr61 (2.60 $⿱$ ) and Asp158 (2.52 $\AA$ ) whereas, no $\pi-\pi$ stacking appeared. Furthermore, compound 4 forms hydrophobic cloud with Met235, Pro66, Val62, Ile159, Ala456, Val455, Val452, Leu451, Tyr215, Ile211, and Met210 (Fig. 4). Both the compounds 3 and 4 showed hydrogen bonds with Arg63 similar to the standard drugs, while compound 3 showed additional hydrogen bond with Thr65 like RO-28-1675 (Fig. 5).

Thus, from the above docked binding mode, it shows that these classes of molecules are good candidates as GK activators and could be further optimized to improve their activity profile.

\section{ADMET evaluation}

All the molecules studied showed a number of hydrogen bond acceptors and hydrogen bond donors less than ten and five, respectively. Also, the number of rotatable bonds in each molecule was less than 10. Topological polar surface area (TPSA) is a very useful descriptor for prediction of transport of drug molecule across the biological barriers and was observed less than $130 \AA^{2}$ in the range of $102.32-105.12 \AA^{2}$. The partition coefficient between $n$-octanol and water $\left(\log P_{o / w}\right)$ is the classical descriptor for hydrophobicity. All the compounds studied were found to have $\log P$ values less than 5 indicating good permeability across the cell membrane. These parameters exhibited that the studied compounds obeyed Lipinski's rule of five [31] with no violation and thus could be good orally active antidiabetic agents.

The solubility of a molecule is a significant property influencing absorption. Also, a water-soluble compound facilitates the ease of formulation and oral administration to deliver a sufficient quantity of active ingredient [29]. All the evaluated compounds were found poorly to moderately water soluble in the range of log scale -4.25 to -6.18 except only compound 3 which showed good solubility in water with a value of -3.76 . The computational data revealed that none of the compounds crosses the blood-brain barrier and hence less possibility to produce neurotoxicity. CYP2D6 belongs to the superfamily of cytochrome P450 (CYP450) which is the most crucial isoenzyme that is responsible for metabolizing most of the drugs in the liver [32]. Inhibition of these enzymes causes drug-drug interaction leading to toxic effects due to accumulation and reduced elimination of drugs or its metabolites [29]. The results revealed that none of the compounds is inhibiting this essential enzyme.

Diabetic patients are at higher risk of developing cardiovascular disease as it is a common diabetesassociated complication [33]. The inhibition of human ether-a-go-go-related gene (hERG) causes prolongation of QT interval leading to fatal ventricular tachyarrhythmia [34]. Compounds 4, 21, and 22 were found to be at a

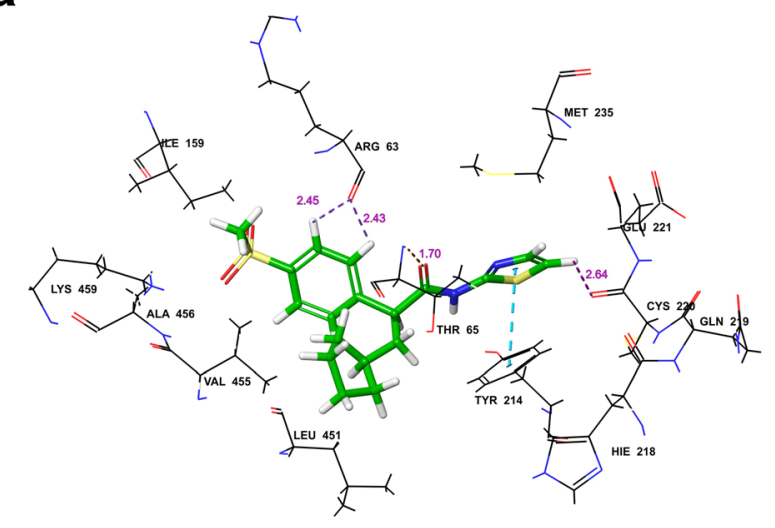

b

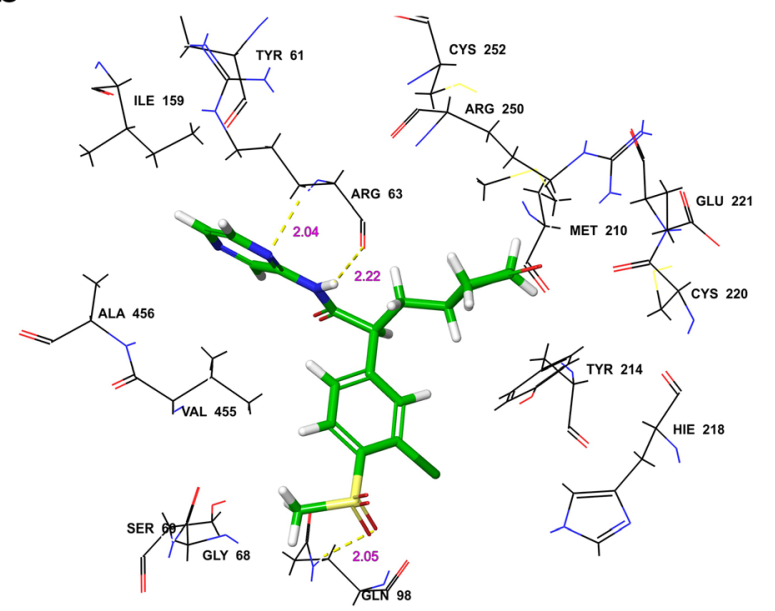

Fig. 5 3D docking poses displaying interaction of (a) RO-28-1675 and (b) piragliatin in the binding site of protein 1V4S 
low-risk of hERG inhibition whereas 3, 5, 6, 12, and 13 at moderate risk. Carcinogenicity is a toxicity that causes cancer in the body. The results specify that except for 6 and 13, all other studied compounds were noncarcinogenic thus stating the safety of compounds in the thriving drug development. Human intestinal absorption (HIA) data are the sum of bioavailability and absorption estimated from the ratio of excretion or cumulative excretion in urine, bile, and feces. The compounds are considered as well absorbed if the absorption is in the range of $70-100 \%$. All the compounds evaluated were found to exhibit high intestinal absorption in the range of 96.86 to 99.56 .

\section{Conclusion}

In the present study, quinazolinone compounds (3-10, 12-19, and 21-28) were designed by excluding reported toxicity producing residues. All compounds were synthesized in satisfactory yield and high purity. Stereoview of the binding pose of ligands at the allosteric site of protein $1 \mathrm{~V} 4 \mathrm{~S}$ revealed crucial hydrogen bonding and $\pi-\pi$ interactions of compounds with amino acid residues. $\mathrm{EC}_{50}$ values obtained by conducting in vitro human GK activation assay exhibited good activity but comparatively less than standards. However, in vivo OGTT assay exhibited a significant reduction in blood glucose in the Wistar rat after glucose challenge. Compounds 3 and 4 were able to produce comparable dock scores and binding free energy in parallel to enzyme and OGTT assay. ADMET studies also revealed that compounds are nontoxic and biologically acceptable. Although the most active derivative in the series (compound 4) showed relatively less activity than the standards, but together, the results we obtained demonstrated our hypothesis of designing GKAs with good efficacy and reduced toxicity.

\section{Abbreviations \\ ADMET: Absorption, distribution, metabolism, excretion, and toxicity; CYP450: Cytochrome P450; $\mathrm{EC}_{50}$ : Half maximal effective concentration; GK: Glucokinase; GKA: Glucokinase activator; hERG: Human ether-a-go-go- related gene; HIA: Human intestinal absorption; LD $_{50}$ : Lethal dose $50 \%$; nM: Nanomolar; OGTT: Oral glucose tolerance test; TPSA: Topological polar surface area}

\section{Acknowledgements}

We are thankful to Dr. S. J. Surana, Principal of R. C. Patel Institute of Pharmaceutical Education and Research, Shirpur for providing research facilities.

\section{Authors' contributions}

We declare that this work was done by the authors named in this article: SCK and NDA conceived and designed the study. SCK and MUD carried out the laboratory work and collected the analytical data. NDA analyzed the spectral, biological, ADME data, and writing of the manuscript. DKL and RRP carried out molecular docking and binding free energy studies. VGU and NBC helped in the evaluation of GK activity and assisted in the data analysis while VAC supervised, revised, and corrected the manuscript. All authors read and approved the final manuscript.
Funding

The authors have no funding to report.

Availability of data and materials

All data and material are available upon request.

Ethics approval and consent to participate

The animal study was approved by the Animal Ethical Committee of the institution under the reference no. IAEC/RCPIPER/2016-17/28.

\section{Consent for publication}

Not applicable

\section{Competing interests}

The authors declare that they have no competing interests.

\section{Author details}

${ }^{1}$ Department of Pharmaceutical Chemistry, R.C. Patel Institute of Pharmaceutical Education and Research, Karvand Naka Shirpur, Dist. Dhule, Maharashtra 425 405, India. ${ }^{2}$ Adv. V. R. Manohar Institute of Diploma in Pharmacy, Wanadongri, Hingna Road, Nagpur 441110, India. ${ }^{3}$ Departamento de Química Orgánica, Facultad de Química y de Farmacia, Pontificia Universidad Católica de Chile, Av. Vicuña McKenna 4860, Macul, 7820436 Santiago, Chile. ${ }^{4}$ Sri Adichunchunagiri College of Pharmacy, Sri Adichunchunagiri University, BG Nagar, Karnataka 571418, India. ${ }^{5}$ Shri Sureshdada Jain College of Pharmacy, Nemi Nagar, Chandwad, Mumbai-Agra Highway, Dist. Nashik, Maharashtra 423 101, India.

Received: 16 October 2019 Accepted: 4 November 2019

Published online: 17 December 2019

\section{References}

1. Matschinsky FM, Zelent B, Doliba N, Li C, Vanderkooi JM, Naji A et al (2011) Glucokinase activators for diabetes therapy: May 2010 status report. Diabetes Care 34:S236-S243. https://doi.org/10.2337/dc11-s236

2. McKerrecher D, Allen JV, Bowker SS, Boyd S, Caulkett PWR, Currie GS et al (2005) Discovery, synthesis and biological evaluation of novel glucokinase activators. Bioorganic Med Chem Lett 15:2103-2106. https://doi.org/10. 1016/j.bmcl.2005.01.087

3. Coghlan M, Leighton B (2008) Glucokinase activators in diabetes management. Expert Opin Investig Drugs 17:145-167. https://doi.org/10. 1517/13543784.17.2.145

4. Guertin K, Grimsby J (2006) Small molecule glucokinase activators as glucose lowering agents: a new paradigm for diabetes therapy. Curr Med Chem 13:1839-1843. https://doi.org/10.2174/092986706777452551

5. Sarabu R, Berthel SJ, Kester RF, Tilley JW (2008) Glucokinase activators as new type 2 diabetes therapeutic agents. Expert Opin Ther Pat 18:759-768. https://doi.org/10.1517/13543776.18.7.759

6. Daniewski AR, Liu W, Radinov RN (2007) Process for the preparation of a glucokinase activator. WO/2007/115968

7. Efanov AM, Barrett DG, Brenner MB, Briggs SL, Delaunois A, Durbin JD et al (2005) A novel glucokinase activator modulates pancreatic islet and hepatocyte function. Endocrinology 146:3696-3701. https://doi.org/10.1210/ en.2005-0377

8. lino T, Tsukahara D, Kamata K, Sasaki K, Ohyama S, Hosaka H et al (2009) Discovery of potent and orally active 3-alkoxy-5-phenoxy- $N$-thiazolyl benzamides as novel allosteric glucokinase activators. Bioorganic Med Chem 17:2733-2743. https://doi.org/10.1016/j.bmc.2009.02.038

9. Bertram LS, Black D, Briner PH, Chatfield R, Cooke A, Fyfe MCT et al (2008) SAR, pharmacokinetics, safety, and efficacy of glucokinase activating 2-(4sulfonylphenyl)-N-thiazol-2-ylacetamides: Discovery of PSN-GK1. J Med Chem 51:4340-4345. https://doi.org/10.1021/jm8003202

10. Mckerrecher D, Allen JV, Caulkett PWR, Donald CS, Fenwick ML, Grange E et al (2006) Design of a potent, soluble glucokinase activator with excellent in vivo efficacy. Bioorganic Med Chem Lett 16:2705-2709. https://doi.org/10. 1016/j.bmcl.2006.02.022

11. Pike KG, Allen JV, Caulkett PWR, Clarke DS, Donald CS, Fenwick ML et al (2011) Design of a potent, soluble glucokinase activator with increased pharmacokinetic half-life. Bioorganic Med Chem Lett 21:3467-3470. https:// doi.org/10.1016/j.bmcl.2011.03.093 
12. Li YQ, Zhang YL, Hu SQ, Wang YL, Song HR, Feng ZQ et al (2011) Design, synthesis and biological evaluation of novel glucokinase activators. Chinese Chem Lett 22:73-76. https://doi.org/10.1016/j.cclet.2010.07.023

13. Cheruvallath ZS, Gwaltney SL, Sabat M, Tang M, Feng J, Wang H et al (2013) Design, synthesis and SAR of novel glucokinase activators. Bioorganic Med Chem Lett 23:2166-2171. https://doi.org/10.1016/j.bmcl.2013.01.093

14. Waring MJ, Brogan IJ, Coghlan M, Johnstone C, Jones HB, Leighton B et al (2011) Overcoming retinoic acid receptor-a based testicular toxicity in the optimisation of glucokinase activators. Medchemcomm 2:771-774. https:// doi.org/10.1039/c1md00090j

15. Grimsby J, Sarabu R, Corbett WL, Haynes NE, Bizzarro FT, Coffey JW et al (2003) Allosteric activators of glucokinase: potential role in diabetes therapy. Science 301:370-373. https://doi.org/10.1126/science.1084073

16. Sarabu R, Bizzarro FT, Corbett WL, Dvorozniak MT, Geng W, Grippo JF et al (2012) Discovery of piragliatin-first glucokinase activator studied in type 2 diabetic patients. J Med Chem 55:7021-7036. https://doi.org/10.1021/ jm3008689

17. Guertin KR (2011) Allosteric activators of Glucokinase (GK) for the treatment of Type 2 diabetes. In: Ward RA, Goldberg F (eds) Kinase Drug Discovery. RSC Publishing, Washington DC, pp 244-261. https://doi.org/10.1039/ 9781849733557-00244

18. Li F, Zhu Q, Zhang Y, Feng Y, Leng Y, Zhang A (2010) Design, synthesis, and pharmacological evaluation of $\mathrm{N}$-(4-mono and 4,5-disubstituted thiazol-2-yl)2-aryl-3-(tetrahydro-2H-pyran-4-yl)propanamides as glucokinase activators. Bioorganic Med Chem 18:3875-3884. https://doi.org/10.1016/j.bmc.2010.04. 038

19. Muñoz ER, Barnett BM (1998) Evaluation of the genotoxicity of aniline. $H C l$ in Drosophila melanogaster. Mutat Res Toxicol Environ Mutagen 413:15-22. https://doi.org/10.1016/S1383-5718(98)00007-2

20. lino T, Sasaki Y, Bamba M, Mitsuya M, Ohno A, Kamata K et al (2009) Discovery and structure-activity relationships of a novel class of quinazoline glucokinase activators. Bioorganic Med Chem Lett 19:5531-5538. https://doi. org/10.1016/.j.bmcl.2009.08.064

21. Taylor EC, Knopf RJ, Borror AL (1960) The dimerization of 2-amino-5nitrobenzonitrile. J Am Chem Soc 82:3152-3157. https://doi.org/10.1021/ ja01497a043

22. Kornblum N, DeLaMare HE (1951) The base catalyzed decomposition of a dialkyl peroxide. J Am Chem Soc 73:880-881. https://doi.org/10.1021/ ja01146a542

23. Nishimura T, lino T, Mitsuya M, Bamba M, Watanabe $H$, Tsukahara D et al (2009) Identification of novel and potent 2-amino benzamide derivatives as allosteric glucokinase activators. Bioorganic Med Chem Lett 19:1357-1360. https://doi.org/10.1016/j.bmcl.2009.01.053

24. Mitsuya M, Kamata K, Bamba M, Watanabe H, Sasaki Y, Sasaki K et al (2009) Discovery of novel 3,6-disubstituted 2-pyridinecarboxamide derivatives as GK activators. Bioorganic Med Chem Lett 19:2718-2721. https://doi.org/10. 1016/j.bmcl.2009.03.137

25. Pfefferkorn JA, Guzman-Perez A, Oates PJ, Litchfield J, Aspnes G, Basak A et al (2011) Designing glucokinase activators with reduced hypoglycemia risk: discovery of N,N-dimethyl-5-(2-methyl-6-((5-methylpyrazin-2-y)carbamoyl)benzofuran-4- yloxy) pyrimidine-2-carboxamide as a clinical candidate for the treatment of type 2 diabetes mellitus. Medchemcomm 2: 828-839. https://doi.org/10.1039/c1 md00116g

26. Kamata K, Mitsuya M, Nishimura T, Eiki Jl, Nagata Y (2004) Structural basis for allosteric regulation of the monomeric allosteric enzyme human glucokinase. Structure 12:429-438. https://doi.org/10.1016/j.str.2004.02.005

27. Lengauer T, Rarey M (1996) Computational methods for biomolecular docking. Curr Opin Struct Biol 6:402-406. https://doi.org/10.1016/S0959440X(96)80061-3

28. Taha MO, Habash M, Khanfar MA (2014) The use of docking-based comparative intermolecular contacts analysis to identify optimal docking conditions within glucokinase and to discover of new GK activators. J Comput Aided Mol Des 28:509-547. https://doi.org/10.1007/s10822-0149740-4

29. Daina A, Michielin O, Zoete V (2017) SwissADME: a free web tool to evaluate pharmacokinetics, drug-likeness and medicinal chemistry friendliness of small molecules. Sci Rep 7:42717. https://doi.org/10.1038/ srep42717

30. Lee SK, Kang Y, Chang GS, Lee IH, Park SH, Park J et al (2017) Bioinformatics and Molecular Design Research Center. Yonsei University, Seoul https:// preadmet.bmdrc.kr
31. Lipinski CA (2004) Lead- and drug-like compounds: The rule-of-five revolution. Drug Discov Today Technol 1:337-341. https://doi.org/10.1016/j. ddtec.2004.11.007

32. Rydberg P, Olsen L (2012) Predicting drug metabolism by cytochrome P450 2C9: comparison with the 2D6 and 3A4 Isoforms. ChemMedChem 7:12021209. https://doi.org/10.1002/cmdc.201200160

33. Aldossari KK (2018) Cardiovascular outcomes and safety with antidiabetic drugs. Int J Health Sci (Qassim) 12:70-83

34. Guth $B D$, Rast $G$ (2010) Dealing with $h E R G$ liabilities early: diverse approaches to an important goal in drug development. Br J Pharmacol 159: 22-24. https://doi.org/10.1111/j.1476-5381.2009.00265.x

\section{Publisher's Note}

Springer Nature remains neutral with regard to jurisdictional claims in published maps and institutional affiliations.

\section{Submit your manuscript to a SpringerOpen ${ }^{\circ}$ journal and benefit from:}

- Convenient online submission

- Rigorous peer review

- Open access: articles freely available online

High visibility within the field

- Retaining the copyright to your article

Submit your next manuscript at $\boldsymbol{\nabla}$ springeropen.com 